\title{
From the Top to the Bottom of the Main Sequence: A Complete Mass Function of the Young Open Cluster M35
}

\author{
David Barrado y Navascués ${ }^{1}$ \\ Max-Planck-Institut für Astronomie, Königstuhl 17, Heidelberg, D-69117 Germany, \\ barrado@pollux.ft.uam.es \\ John R. Stauffer ${ }^{2,3}$ \\ Harvard-Smithsonian Center for Astrophysics, 60 Garden St., Cambridge, MA 02138, USA, \\ jstauffer@cfa.harvard.edu \\ Jerôme Bouvier ${ }^{3}$ \\ Laboratoire d'Astrophysique, Observatoire de Grenoble, Université Joseph Fourier, B.P. 53, 38041 \\ Grenoble Cedex 9, France, jbouvier@obs.ujf-grenoble.fr \\ and \\ Eduardo L. Martín 3,4 \\ California Institute of Technology, MS 150-21, Pasadena, CA 91125, USA ege@gps.caltech.edu
}

\begin{abstract}
We present very deep and accurate photometry of the open cluster M35. We have observed this association in the Cousins R,I filters, together with the Johnson V filter. We have covered a region of $27.5 \times 27.5$ square arcmin, equivalent to a fifth of the total area of the cluster. The data range from $\mathrm{I}_{\mathrm{c}}=12.5$ to 23.5 magnitudes, and the color intervals are $0.4 \leq(\mathrm{V}-\mathrm{I})_{\mathrm{c}} \leq 3.0,0.5 \leq(\mathrm{R}-\mathrm{I})_{\mathrm{c}} \leq 2.5$. Roughly, these values span from $1.6 \mathrm{M}_{\odot}$ down to the substellar limit, in the case of cluster members. By using the location of the stars on color-magnitude and color-color diagrams, we have selected candidate members of this cluster. We have merged our sample with previously published data and obtained a color-magnitude diagram for the complete stellar population of the cluster,
\end{abstract}

\footnotetext{
${ }^{1}$ Present address: Departamento de Física Teórica, C-XI. Universidad Autónoma de Madrid, Cantoblanco, E28049 Madrid, Spain

${ }^{2}$ Visiting Astronomer Kitt Peak National Observatory. KPNO is operated by AURA, Inc., under contract to the National Science Foundation.

${ }^{3}$ Visiting Astronomer, Canada-France-Hawaii Telescope

${ }^{4}$ Present address: Institute for Astronomy, University of Hawaii, Honolulu, HI96822, USA
} 
covering the spectral range early B - mid M. Based on the distribution of field and cluster stars in color-magnitude and color-color diagrams, we estimate that two thirds of these candidates are likely to be true members of M35. These stars approximately double the number of stars identified as candidate members of this cluster $(\sim 2700)$. We provide the photometry and accurate positions of these stars. The deep photometry has allowed us to study the mass segregation within the cluster, the Luminosity Function and Mass Function. We show that in the magnitude range $13 \leq \mathrm{I}_{c} \leq 22$ there is a reduced mass segregation, in opposition to what happens to higher mass stars, where the mass segregation is stronger. The Luminosity Function behaves essentially as the one characteristic of the Pleiades, presenting a peak at $\mathrm{I}_{\mathrm{c}} \sim 19$ magnitudes $\left(\mathrm{M}_{\mathrm{I}} \sim 9\right)$. Combining our photometry with previous data corresponding to more massive stars, we find that the Mass Function increases monotonically, when plotted in a log-log form, until it reaches $\sim 0.8 \mathrm{M}_{\odot}(\alpha=2.59)$. It remains shallower for less massive stars $(\alpha=0.81$ for $0.8-0.2 \mathrm{M}_{\odot}$ ), whereas a decrease ins observed for stars close to the substellar regime. These different behaviors suggest that at least three mechanisms play a role in the formation of stellar and substellar objects. The total mass of the cluster is $\sim 1600 \mathrm{M}_{\odot}$ in the area covered by this study.

Subject headings: open clusters and associations: M 35, NGC 2168; photometry; late spectral type stars; stellar mass function

\section{Introduction}

Open clusters serve as Rosetta stones to study stellar properties because they provide homogeneous samples of known age, metallicity and distance. Therefore, open clusters can be used to determine empirically the evolution of different stellar properties such as the activity, rotation, chemical abundances, mass loss, et cetera. For low mass stars, however, only a handful of open clusters have been studied systematically, essentially due to the fact that most clusters are far away, with distance moduli ranging from 6 to 12 magnitudes. With the recent improvements in detectors and with the availability of large ground-based telescopes, it is possible to obtain high quality data for a larger number of clusters.

M35 (NGC 2168) is a very interesting open cluster for several reasons. It is moderately nearby, with $(\mathrm{m}-\mathrm{M})_{0}=9.7$ (Vidal 1973). It is very well populated, containing several thousand members with a total dynamical mass estimated between 1600 and $3200 \mathrm{M}_{\odot}$ (Leonard \& Merritt 1989). It is spread over an area of $\sim 1 \times 1$ square degrees, making it a suitable target for multifiber spectroscopy. The contamination by field stars is not overwhelming, as happens in some other clusters of similar characteristics. The reddening is also moderate $-\mathrm{E}(\mathrm{B}-\mathrm{V})=0.17-0.225$. Perhaps one of the most interesting properties is its youth, since it is said to be a cluster coeval with the Pleiades, based

on the fact that their upper-main sequence turn-off locations are said to be at the same position in 
color-magnitude diagrams (Vidal 1973).

Until very recently, only the upper-main sequence (MS) of M35 had been studied with some detail. Photometric (UBV filters) and/or proper motion searches were carried out by Cuffey (1938), Hoag et al. (1961), Cudworth (1971), Vidal (1973), McNamara \& Sekiguchi (1986a). They reached barely $\mathrm{V}=15$, about $1.2 \mathrm{M}_{\odot}$, in the case of M35 members. At the present time, different groups have conducted several multi-band CCD photometry which reach much deeper members, such as Sung \& Bessell (1999=SB1999), von Hippel et al. (2000) and Sarrazine et al. (2000). The last works present data in the UBVRI filters, reaching beyond V=19, whereas Sung \& Bessell (1999) used the UBVI passbands only, reaching one magnitude fainter. Sung \& Bessell (1999) were able to estimate the interstellar reddening, the distance and the age of the cluster, deriving $\mathrm{E}(\mathrm{B}-\mathrm{V})=0.255 \pm 0.024$, $(\mathrm{m}-\mathrm{M})_{0}=9.60 \pm 0.10$ and $\tau=200_{-100}^{+200}$ Myr. Previously, Sung \& Lee (1992) derived a distance modulus $(\mathrm{m}-\mathrm{M})_{0}=9.3$, an age $\tau=85 \mathrm{Myr}$, and a differential internal extinction $\mathrm{E}(\mathrm{B}-\mathrm{V})=0.26-0.44$. A different estimate of the distance and reddening have been published by von Hippel et al. (2000), E(B$\mathrm{V})=0.3,(\mathrm{~m}-\mathrm{M})_{V}=10.25$. These authors also estimated an age by fitting isochrones, yielding $\tau=100$ Myr. However, they inferred that M35 is older than the Pleiades by comparing in a differential way members of both clusters. Based on an extension of the data presented by von Hippel et al. (2000), and assuming a metallicity of $[\mathrm{Fe} / \mathrm{H}]=-0.21$ (Barrado y Navascués et al. 2000a,b), Sarrazine et al. (2000) derived $(\mathrm{m}-\mathrm{M})=10.16 \pm 0.10, \mathrm{E}(\mathrm{B}-\mathrm{V})=0.198 \pm 0.008$ and $\tau=160 \pm 40 \mathrm{Myr}$. This age and the Sung \& Bessell (1999) value are significantly older than the canonical upper-main sequence age of the Pleiades, 70-100 Myr (Mermilliod 1981; Meynet, Mermilliod, \& Maeder 1993). These older ages are in agreement with our own findings concerning the distribution of rotational velocities and lithium abundances (Barrado y Navascués et al. 2000a, 2000b). Based on all the available information, they assumed an age of $\tau=175$ Myr. Note that Barrado y Navascués et al. (2000b) have derived the first metallicity estimate for M35 based on high resolution spectroscopy, with the resulting value of $[\mathrm{Fe} / \mathrm{H}]=-0.21 \pm 0.10$, lower than the value corresponding to the Pleiades, $[\mathrm{Fe} / \mathrm{H}]=+0.01$, estimated in the same fashion by these studies. The Pleiades cluster is one of the cornerstones of astrophysics, and the comparison of members of both clusters can provide additional information for understanding their properties. Although several stellar characteristics are thought to depend essentially on age (rotation, coronal and chromospheric activity, et cetera), this assumption has been recently challenged by Randich \& Schmitt (1995), comparing the X-ray luminosities of Praesepe and the Hyades, two coeval clusters (see also the discussion in Barrado y Navascués et al. 1997, 1998a). Extensive and detailed studies of several clusters of similar age can help to understand this crucial problem, as well to clarify the evolution of the stellar properties when comparing clusters of different ages.

We have embarked on an ambitious study of the open cluster M35, combining high resolution spectroscopy to determine lithium abundances, radial and rotational velocities (Barrado y Navascués et al. 2000a, 2000b) with deep imaging to extend the membership list to nearly the hydrogen burning mass limit (or beyond) and estimate the cluster Mass Function (Barrado y Navascués et al. 1998b). The current paper is devoted to the presentation of our deep imaging 
data, to study the complete MF, the mass segregation within the cluster and to estimate the total mass of M35.

\section{Observations}

We have carried out photometric observations of the young cluster M35 in two different campaigns. The first run took place in December 11th, 1995, at the $\mathrm{KPNO}^{5} 4 \mathrm{~m}$ telescope. In this case, we used the prime focus, a CCD camera, with a Tek $2048 \times 2048$ pixels, yielding a field of $23.2 \times 23.2$ square arcmin. For the second run we used the Canada-France-Hawaii Telescope (December 13th, 1996) and the mosaic camera $(8000 \times 8000$ pixels $)$, which provided a field of $27.5 \times 27.5$ square arcmin.

The observations were processed using standard procedures (bias subtraction, flat-field correction, etc). We used the $\mathrm{IRAF}^{6}$ package in the case of the KPNO data. Since our aim was to obtain photometry of cool stars over a very large dynamical range, we collected two sets of images of different exposure times during the KPNO run, namely 60 and $600 \mathrm{sec}$ for the V filter, and 10 and $120 \mathrm{sec}$ for the Cousins $\mathrm{I}_{\mathrm{c}}$ band (Cousins 1978). Therefore, we reached $\left(\mathrm{V}, \mathrm{I}_{\mathrm{c}}\right)_{\text {limit }}=(20.2,18.2)$ for the shallow set and $\left(\mathrm{V}, \mathrm{I}_{\mathrm{c}}\right)_{\text {limit }}=(21.1,19.5)$ for the deep exposures. The completeness limits are $\mathrm{V}_{\text {complete }}=19.0$ and $\mathrm{V}_{\text {complete }}=19.75$, respectively. In the case of the CFHT survey, we collected data in the magnitude interval $17.5 \leq \mathrm{I}_{\mathrm{c}} \leq 23.5,0.5 \leq(\mathrm{R}-\mathrm{I})_{\mathrm{c}} \leq 2.5$. The completeness limit was $\mathrm{I}_{c, \text { complete }}=22.1$ magnitudes in this case. Errors can be estimated as 0.05 magnitudes for each filter at the bright end, degrading to 0.15 magnitudes for the faintest objects in each dataset.

For the KPNO observations, the calibration of the data was performed by observing standard stars in Landolt (1992) -SA 92, 94, 95, 98, 100, 112, and 113- several times during the observing run. We derived our own extinction coefficients, yielding $\mathrm{C}_{\mathrm{V}}=0.192 \pm 0.006$ and $\mathrm{C}_{\mathrm{I}}=0.110 \pm 0.003$. The calibrated data was obtained by using the expressions:

$$
\begin{aligned}
& V=v-3.583-0.192 \times \text { airmass }-0.046 \times(v-i) \\
& I=i-4.276-0.110 \times \text { airmass }+0.012 \times(v-i)
\end{aligned}
$$

where V,I denote the calibrated magnitudes, whereas v,i refer to the instrumental values.

We have compared our final calibrated data with the two previous studies which are deep enough. The comparison between our data and the values from Sung \& Bessell (1999) indicates

\footnotetext{
${ }^{5}$ Kitt Peak National Observatory is operated by AURA, Inc., Association of Universities to research in Astronomy, under contract to the National Science Foundation.

${ }^{6}$ IRAF is distributed by National Optical Astronomy Observatories
} 
that the I data are essentially identical, whereas there is a difference in the data corresponding to the $\mathrm{V}$, in the sense $\mathrm{V}(\mathrm{SB} 1999)=\mathrm{V}$ (this work $)+0.03$ magnitudes. Of this difference, 0.01 magnitudes can be attributed to the difference between the Landolt (1992) photometry (the values used for the calibration), and the SAAO photometry, which defines the Cousins system. Regardless of this systematic difference, our final results (membership selection and Mass Function analysis) are not affected by it.

The CFHT observations were carried out and analyzed in the same fashion as our observations of the Pleiades and Praesepe (Bouvier et al. 1998). In all cases the data were recorded under photometric conditions and the calibration was performed independently from each other using standard stars listed in Landolt (1992). Several very red standard stars were observed to ensure the accurate transformation from the instrumental system into the Cousins system. Visual inspection of the stars in common to the two set of data (KPNO and CFHT), as listed in Table 2, indicates that the calibrated magnitudes are in excellent agreement.

In the case of the KPNO survey, coordinates were derived for all the detections using the GSC catalog. Positional accuracies are better than 0.5 arcsec. An empirical confirmation for the accuracy of the coordinates was provided by the good quality of the spectra we obtained using our coordinates for a WIYN/HYDRA multi-object spectroscopy run (Barrado y Navascués et al. 2000a, 2000b).

\section{The Selection of Members}

\subsection{A Main Sequence for Cool Stars}

In order to select possible M35 cluster members based on their location in a color-magnitude diagram (CMD), we need to have a reliable main sequence locus for low mass stars. Theoretical isochrones (e.g. Baraffe et al. 1998) do not yet do this optimally for optical colors due to remaining problems with cool dwarf model atmospheres and inadequate detailed line opacities used to compute fluxes. Therefore, we must rely on an empirical main sequence. We have constructed one using field stars from Leggett (1992) which fulfill two conditions: they have accurate parallaxes (П), in the sense $\sigma(\Pi) / \Pi \leq 0.10$, and they were not listed as "AB" in that paper (that is, they are not spectroscopic binaries or close visual binaries). The selected data come from Luyten (1972), Giclas et al. (1971), Gliese (1969) and Gliese \& Jahreiss (1979) -see reference therein for the original sources of the photometric data-. Figure 2 of Bouvier et al. (1998) and Figure 1 of Stauffer et al. (1999) show this empirical MS when applied to the Pleiades and Alpha Per open clusters, respectively, in the $\left[\mathrm{I}_{\mathrm{c}},(\mathrm{R}-\mathrm{I})_{\mathrm{c}}\right]$ plane. Figure 1 of Barrado y Navascués et al. (1999) displays the comparison between the empirical MS and field stars from the Leggett $(1992)$ in the $\left[\mathrm{V},(\mathrm{V}-\mathrm{I})_{\mathrm{c}}\right]$ plane. As can be seen, the lower envelope is an appropriate MS.

For the current paper, we need to extend this MS to bluer (higher mass) stars. We have done 
this first by tracing a lower envelope to M35 members identified by Sung and Bessell (1999) in the $\mathrm{I}_{\mathrm{c}}$ magnitude range 8.5 to 14.0. Second, we used accurate radial velocities for 78 of our photometric candidates down to $\mathrm{I}_{\mathrm{c}}=16$, to determine whether they are indeed members (Barrado y Navascués et al. 2000b, shown as solid circles). The loci of those bona fide members were used to extend the Sung \& Bessell (1999) data and to connect it with the empirical isochrone derived from Leggett's data. The final result can be seen in Figure 1a and Figure 1b. Note that for the lowest masses, we would expect M35 members to be above (to the right) of this curve because the M35 members will still be contracting to the main sequence, assuming the canonical age of the Pleiades. However, the low metallicity of M35 $-[\mathrm{Fe} / \mathrm{H}]=-0.21-$, produces a shift of the location of its members compared similar stars of solar composition and as discussed in Section 1, there are evidences that M35 is older than the Pleiades. At higher masses, our MS curve should serve as an approximate lower envelope to the distribution of M35 members (e.g. photometric binaries should still scatter up to 0.75 mag above the curve). Our empirical MS can be found in Table 1.

\subsection{Color-magnitude and color-color diagrams}

\subsubsection{KPNO Data}

Figures $1 \mathrm{a}$ and $1 \mathrm{~b}$ depict the photometric $\mathrm{VI}_{\mathrm{c}}$ data collected at KPNO. The first panel shows the CMD for the shallow exposure, whereas panel b contains the values for the deep one. In both cases, limiting and completeness magnitudes are indicated as solid and dashed lines. Our empirical MS sequence is also shown as a solid line. We used a distance modulus of $(\mathrm{m}-\mathrm{M})_{0}=9.60$, a color excess of $\mathrm{E}(\mathrm{V}-\mathrm{I})_{\mathrm{c}}=0.321$ and an interstellar absorption of $\mathrm{A}_{\mathrm{V}}=0.765\left(\mathrm{R}_{V}=3.0\right)$, which corresponds to the reddening estimated by Sung \& Bessell (1999) of $\mathrm{E}(\mathrm{B}-\mathrm{V})=0.255$. The probable members of M35 (Barrado y Navascués et al. 2000b), observed spectroscopically ( $<\mathrm{RV}>=-8 \mathrm{~km} / \mathrm{s}$ ), are shown as solid circles.

We have selected as initial possible candidate members of M35 all those stars which have photometry in a strip described by the empirical MS and the MS minus 0.75 magnitudes (to allow for binarity) and \pm 0.1 magnitudes, to allow for photometric errors and errors in the distance and the reddening. Hence, the strip is 0.95 magnitudes wide. Another example of photometric selection of candidate members of a cluster can be found in Barrado \& Byrne (1995), in the case NGC5460, and Barrado y Navascués et al. (2000c) for IC2391. The selection of M35 candidate members was stopped at $\mathrm{V}=21.0$ magnitudes. Therefore, we have covered the spectral range F0-K7 for cluster members. Note that despite the lack of separation between field stars and the locus of the M35

main sequence, the diagrams show a relative concentration of objects corresponding to M35, very clear in Figure 1b in the range $1.5 \leq(\mathrm{V}-\mathrm{I})_{\mathrm{c}} \leq 2.0$. 


\subsubsection{CFHT Data}

Our initial optical photometric survey for members of M35 was extended by taking longer exposures at the Canadian-French-Hawaii telescope. In this case, we obtained 2 exposures in the Cousins $R$ and I filters. Figure $1 c$ displays the $I_{c}$ versus $(R-I)_{c}$ color-magnitude diagram. The objects detected by this survey are plotted as dots. We have added the empirical MS for field stars (see section 3.1), shifted according to the reddening $-\mathrm{E}(\mathrm{R}-\mathrm{I})_{\mathrm{c}}=0.178$ - and the distance modulus $-(\mathrm{m}-\mathrm{M})_{\mathrm{I}}=10.044-$ of M35 (see previous subsection). Note that the MS really traces the relative concentration of very cool stars in M35, although there is a shift of $\sim 0.1$ magnitudes, which can be attributed to the low metallicity of the cluster. Note that the lower, redder end of the CMD corresponds to objects of M5.5 spectral type. The location of the M35 low mass candidate members, below our empirical MS, seems to indicate that they are not pre-MS objects, and therefore, they are older than $\sim 100 \mathrm{Myr}$, in agreement with the age by Sung \& Bessell (1999) and Sarrazine et al. (2000) and our previous findings (Barrado y Navascués et al. 2000b).

Figure 1c clearly shows two groups of stars: A significant fraction of the stars are on the locus of M35. Most of the detected stars lie between $0.5<(\mathrm{R}-\mathrm{I})_{\mathrm{c}}<1.7$, following a distribution parallel to the M35 MS, but 4 magnitudes fainter. However, there is not a clear gap, empty of stars, between the former (M35 candidate members) and the latter groups (field stars). A smooth transition is present, indicating that any list of candidates of M35 presents contamination by field stars. In any case, the diagram allows us to perform a tentative identification of candidates for membership to the cluster. We have identified candidate members of M35 here in the same way that we did for the KPNO data, except in this case allowing also for the fact that the faintest M35 members will still be contracting to the MS, and for uncertainties in our empirical MS, as well as in the cluster age, distance and reddening. We have cross-correlated the list of stars selected from the $\mathrm{VI}_{\mathrm{c}}$ plane with the list coming from the $\mathrm{RI}_{\mathrm{c}}$ survey, merging both sets of data. Note that the intersecting magnitude range is only one magnitude wide, from 17.5 to 18.5 in $\mathrm{I}_{\mathrm{c}}$.

\subsubsection{The $(R-I)_{\mathrm{c}}-(V-I)_{\mathrm{c}}$ Plane}

Our two surveys (CFHT and KPNO) overlap over a range of one magnitude. Therefore, we have merged both datasets, generating a subsample of data with measurements in three passbands. Figure 2 shows $(\mathrm{R}-\mathrm{I})_{\mathrm{c}}$ against $(\mathrm{V}-\mathrm{I})_{\mathrm{c}}$ for this subgroup. We have included in the figure the average behavior for field stars, after Leggett (1992) and Bessell (1991), which are shown as solid and dashed lines, respectively. Very late spectral type members of the young clusters IC2391 (Barrado y Navascués et al. 2000c) are also included as a comparison group. Based on this diagram, we have rejected several stars as members of the cluster, due to their location (cross symbols). The solid dots represents the photometric members of the clusters. 


\subsubsection{A List of Members}

Our list of members contains 1945 stars, with 275 stars previously observed (essentially by McNamara \& Sekiguchi 1986a and Sung \& Bessell 1999). Therefore, we present a list with 1670 new possible photometric members of the cluster, doubling the number of candidate members of the cluster. Including all previously known members of M35, there are in total about 2710 identified candidate members. As a comparison, the Pleiades has 1194 known members (Pinfield et al. 1998).

Table 2 lists our identifying number for each object and the photometry in columns \#1 to \#6. Positions are listed in columns \#7 and \#8. Finally, cross identification with WEBDA database (Mermilliod 1996), McNamara \& Sekiguchi (1986a) and Sung \& Bessell (1999), and membership probability, derived from the proper motion, are included from column \#9 to column \#12. Note that Mermilliod's identification number in his WEBDA database coincide with the identification number of Cuffey (1938) for the first 778 stars. Stars in the M35 field of view in WEBDA database are identified with numbers from 1 to 4219 (the database includes 1566 entries, some of them are in fact no members). We have decided to identify our M35 candidate members with numbers starting at 5001.

Our final membership assignment is also listed in Table 2. Those stars with radial velocity and/or with proper motions in agreement with the cluster average are flagged with "Y+" in column \#13. The same criteria were used to reject several candidates and they have the "N+" flag. Candidate members with positions in the color-color diagram which do not correspond to the cluster locus appear as " $\mathrm{N}$ ", whereas those stars which only have $\mathrm{V}, \mathrm{I}_{\mathrm{c}}$ and/or $\mathrm{R}_{\mathrm{c}}, \mathrm{I}_{\mathrm{c}}$ data are listed as "Y".

Final color-magnitude diagrams, in absolute $\mathrm{I}_{\mathrm{c}}$ magnitude and dereddened colors, are presented in Figure 3a, Figure 3b and Figure 3c. In all three cases, our empirical main sequence is displayed as a solid line. In Figure 3a, data from the literature (Sung \& Bessell 1999) appear as asterisk symbols (membership probability, from the proper motions, larger than 0.80, "Y+"). Our bona fide members, selected from their radial velocity (Barrado y Navascués et al. 2000b), are shown as solid circles ("Y+"). Other photometric members are displayed as open circles ("Y"), whereas nonmembers appear as crosses ("N", "N+"). Figure 3b displays the photometric candidate members from the CFHT survey $-\mathrm{I}_{\mathrm{c}}$ versus $(\mathrm{R}-\mathrm{I})_{\mathrm{c}}$. A complete color-magnitude diagram for all bona fide and candidate members of M35 is shown in Figure 3c, stretching from $\mathrm{M}\left(\mathrm{I}_{\mathrm{c}}\right)=-1.5$ up to $\mathrm{M}\left(\mathrm{I}_{\mathrm{c}}\right)=13.0$, and covering from early B down to M5.5 spectral types. It includes our own candidate members and those candidate members from Sung \& Bessell (1999) which are probable members, based on their proper motions, as derived from McNamara \& Sekiguchi (1986a). Note that in order to show our CFHT data at the red, faint end, we derived $(\mathrm{V}-\mathrm{I})_{\mathrm{c}}$ colors from $(\mathrm{R}-\mathrm{I})_{\mathrm{c}}$ and our empirical MS. The star HD41996, which is a likely member based on its proper motion and has already evolved off the MS, is out of the limits of the figure. 


\subsubsection{Brown Dwarfs in the cluster}

Our fainter candidates reach $\mathrm{I}_{\mathrm{c}} \sim 23.0$, which corresponds to $\mathrm{M}\left(\mathrm{I}_{\mathrm{c}}\right) \sim 13.0$ magnitudes. Following Stauffer, Schultz \& Kirkpatrick (1998) and the theoretical models of Baraffe (1998, priv. communication), the substellar limit should be, for an age of $125-200 \mathrm{Myr}$, at $\mathrm{I}_{\mathrm{c}}(\mathrm{BD}) \simeq 22.1-22.6$, and the lithium depletion boundary (see Stauffer \& Barrado y Navascués 2000) at $\mathrm{I}_{\mathrm{c}}(\mathrm{LDB}) \simeq 22.3-23.2$ magnitudes. Therefore, we have reached the substellar domain in M35, and found a sample of the farthest brown dwarf (BD) candidates discovered so far. In total, we have 65 objects with masses in the range $0.075-0.055 \mathrm{M}_{\odot}$, based on a $100 \mathrm{Myr}$ isochrone from D'Antona \& Mazzitelli (1994). Note that if the masses of cluster brown dwarfs sum up about a few percent of the total mass of the association, it would mean that M35 has a minimum of 600-1000 substellar objects. For instance, brown dwarf searches in the Pleiades have estimate that the total contribution of BD to the mass of the cluster can be 5\% (Bouvier et al. 1998) or 3\% (Hodgkin \& Jameson 2000).

\subsection{Contamination by field stars}

The relatively smooth transition between the concentration of field stars, fainter and/or bluer than M35, and this cluster, indicates that our list of member candidates is contaminated by field stars. However, note that in the case of the $\mathrm{V},(\mathrm{V}-\mathrm{I})_{\mathrm{c}}$ diagram, the gap between the M35 main sequence and the bulk of the field stars increase with the magnitude: the separation in color is smaller at the upper part of the diagram and, therefore, the number of spurious members should be larger for stars of spectral type G and early K. Moreover, the separation in color between the field stars and the cluster locus is larger in the case of the $\mathrm{I},(\mathrm{R}-\mathrm{I})_{\mathrm{c}}$ diagram, a fact which indicates that it is easier to estimate the contamination by field stars in this case than from the $\mathrm{V},(\mathrm{V}-\mathrm{I})_{\mathrm{c}}$ diagram.

In the case of the brighter stars presented in this work, which come from the $\mathrm{V},(\mathrm{V}-\mathrm{I})_{\mathrm{c}} \mathrm{CMD}$, we have estimated the contamination by field stars using two different methods: (i) Some of the stars have been discovered previously, and proper motions and their associated membership probability are listed by the authors (Cudworth 1971; McNamara \& Sekiguchi 1986a). They have magnitudes in the range $13.7 \leq \mathrm{V} \leq 14.9$, which correspond to F2-G2 spectral types. We have compared the number of stars present in our survey having probability less than $50 \%$ with the total number, and assumed that this ratio is the contamination rate. The result is $37 \%$. If a more demanding criterion is selected (such as membership probability less than $80 \%$ ), the pollution rate does not change much $(53 \%)$. (ii) We have previously derived radial velocities for a subsample of these stars (Barrado y Navascués et al. 2000b). They have magnitudes in the range $14.5 \leq \mathrm{V} \leq 17.5$, corresponding to F9-K5 spectral types. We have used the radial velocity as a membership criterion. The pollution rate derived from the comparison between the bon a fide members and non-members is $30 \%$. These

numbers confirm our initial conclusion that contamination is stronger in the upper Main Sequence (for $\mathrm{F}$ and $\mathrm{G}$ stars) than for $\mathrm{K}$ members. 
In the case of the deep survey from CFHT, we have divided the $\mathrm{I}_{c},(\mathrm{R}-\mathrm{I})_{c}$ color-magnitude diagram in boxes of size 0.5 magnitude by 0.1 magnitude in $\mathrm{I}_{\mathrm{c}}$ and $(\mathrm{R}-\mathrm{I})_{\mathrm{c}}$, respectively. We have constructed diagrams representing the number of stars in each $i$ box $-\mathrm{N}_{i, *^{-}}$against $(\mathrm{R}-\mathrm{I})_{\mathrm{c}}$ for a given $\mathrm{I}_{\mathrm{c}}$ interval. Figure 4 shows one such diagram, corresponding to the interval $18.0 \leq \mathrm{I}_{\mathrm{c}}<18.5$ magnitudes. The numbers of stars are shown as solid circles. Two peaks are apparent, one near $(\mathrm{R}-\mathrm{I})_{\mathrm{c}}=0.65$ and the other close to $(\mathrm{R}-\mathrm{I})_{\mathrm{c}}=1.35$, the last one corresponding to the location of M35. Then, we have counted the number of stars in the box corresponding to the M35 peak, the box immediately bluer, and the two redder boxes, delineated in Figure 4 by the horizontal line segment. The total number of stars in all four boxes, $\mathrm{N}_{i, *}\left(\mathrm{I}_{\mathrm{c}}\right)$, is the initial number of members of M35 in the considered interval of $\mathrm{I}_{\mathrm{c}}$. In order to remove the contamination due to field stars, we have fitted three Gaussian curves to $\mathrm{N}_{i, *}\left(\mathrm{I}_{\mathrm{c}}\right)$, one corresponding to the bulk of the field stars, the other to M35, and the third to the transition between the former two. There is no physical reason for this procedure, but the shape of any particular peak is pseudo-Gaussian, and we know that $\mathrm{N}_{i, *}$-the number of stars in each box- has to be null on both sides of Figure 4 or the equivalent in other $\mathrm{I}_{\mathrm{c}}$ ranges. The computed distribution of field stars is shown as a dashed line in the figure. Note the excellent agreement between the data and the fit for the field stars. We have subtracted the computed number of field stars for each box from the measured number, for the boxes corresponding to the location of M35. This value, $\mathrm{N}_{i, *}^{\mathrm{M} 35}\left(\mathrm{I}_{\mathrm{c}}\right)$, is the number of cluster members and is listed in column \#5 of Table 3 (in the case of the CFHT data).

Table 3 contains the ratio between the spurious members of the cluster and the total total number of stars - members+field stars-, per bin 0.5 magnitude wide in $\mathrm{I}_{\mathrm{c}}$ (column \#4). This is a preliminary result, and only more accurate methods, such as proper motion measurements or accurate radial velocities, can guarantee the membership of a particular star or group of stars.

\section{Mass segregation in the center of the cluster}

Stellar clusters are not stable from the gravitational point of view. They disipate as they orbit around the Galaxy, due to the gravitational interaction with the spiral arms, interstellar clouds, other clusters, et cetera. In addition, since they behave as a N-body problem, they try to find gravitational equilibrium by expelling members, which tend to be the less massive, while the most massive stars move toward the center. Therefore, mass segregation appears in relaxed clusters.

M35 has an angular diameter of, at least, 33 arcmin, since proper motion members have been detected at that distance (Cudworth 1971). In fact, Leonard \& and Merritt (1989) stated that the tidal radius could reach between 33 and 66 arcmin, extending the cluster even further. Our survey covers an area of $27.6 \times 27.6$ arcmin, a significant fraction of the total area in the core. In this central part of the cluster, we have found strong evidence of a limited mass segregation, in a specific way. This mass segregation appears when we compare our sample with the sample of more massive stars from McNamara \& Sekiguchi (1986a). McNamara \& Sekiguchi (1986b) found that essentially almost $100 \%$ of the proper motion members are within an angular radius of 20 
arcmin, regardless of the mass $\left(6.0<\right.$ Mass $\left.<1.2 \mathrm{M}_{\odot}\right)$, based on data from Cudworth (1971). They also established that there is mass segregation in this mass range, using either Cudworth's or their own data (see their Figure 1). Similar conclusion has been reached by von Hippel et al. (2000), for stars brighter than V=19 magnitudes. However, our data, composed of less massive stars, are distributed homogeneously on the surveyed sky section, without any clear decrease in the number of stars toward the borders, indicating that the distribution of low mass stars could be extended far beyond the 20 arcmin radius.

To illustrate this situation further, Figure 5 displays the cumulative distribution of stars versus the angular distance (in arcmin, bottom of the diagram) or radius (in parsecs, top of the diagram). Open circles represent the stars from Sung \& Bessell (1999) which have $\mathrm{I}_{c} \leq 13$ magnitudes, whereas solid and open triangles represent data from our survey $\left(13<\mathrm{I}_{c} \leq 17.5\right.$ and $17.5<\mathrm{I}_{c} \leq 21.5$, respectively). The mass segregation for the brighter members seems obvious. However, the two fainter subsamples resemble each other, except in the interval 3.5-7 arcmin, where a small difference appears. Moreover, the distributions in the intervals $\mathrm{I}_{c}=17.5-19.5$ and $\mathrm{I}_{c}=19.5-21.5$ are almost identical. However, some mass segregation clould be present at the very bottom of the MS, around the substellar limit (see Subsection 5.2.4). These facts indicate that the cluster is not fully relaxed.

\section{Luminosity and Mass Functions}

\subsection{The Luminosity Function}

Figure 6 shows the Luminosity Function of M35, once the spurious members were removed. Solid circles represent data coming from the KPNO survey, whereas open circles correspond to the fainter data, collected at CFHT. We have included the errors as vertical bars (see section 5.1.1). Note that apparent magnitudes are indicated with the bottom of the $\mathrm{x}$-axis, whereas absolute magnitudes appear at the top. The distribution peaks around $I_{c}=19$, as happens in the Pleiades (Zapatero-Osorio 1997). Fainter than this magnitude, the number of stars decreases with magnitude and a strong contamination by field stars appears. Our sample is complete up to $\mathrm{I}_{\mathrm{c}}=22.0$ magnitudes. Therefore, the last point is uncertain and represents only an upper limit. We have not considered the effect of binarity when computing the LF (or Mass Function).

There is an additional remark regarding the position of M35 in the CMD. We located the ZAMS in the diagram using the published values in the literature and appropriate conversions to

the photometric bands we have used. However, we did not use this information when counting the number of stars in M35. Therefore, our method is totally independent of distance modulus and reddening. However, we have obtained that the maximum density of stars, given a $(\mathrm{R}-\mathrm{I})_{\mathrm{c}}$ value, appears $\sim 0.1$ magnitudes below the ZAMS, as a visual inspection indicates in Figure 3b. This seems to be an effect of the low metallicity of M35. 


\subsubsection{Estimation of the errors}

To estimate the uncertainties in the Luminosity Function, we took into account the error in the photometry and those produced by the way we removed the contamination by field stars. The error in $\mathrm{I}_{\mathrm{C}}$ is $0.05 \mathrm{mag}$. Since the bin size is 0.5 magnitudes, $20 \%$ of the number of stars $-\mathrm{N}_{i, *}\left(\mathrm{I}_{\mathrm{C}}\right)-$ in a particular $i$ box could be assigned to a nearby box (i+1 or $i-1)$. In addition, we only took into account the four boxes around the location of M35 (two redder and one bluer, in addition to the box located at the position of the cluster MS). The error in the removal of the field stars, $\Delta \mathrm{N}_{\text {field }}$, can be estimated as the square root of the sum of the the number of stars in the external boxes

delimiting the cluster, $\mathrm{N}_{i, *}^{\mathrm{inf}}$ and $\mathrm{N}_{i, *}^{\mathrm{sup}}$ in the case of the faint stars or as $30-40 \%$ for the brighter stars of our sample. Therefore, the total error is:

$$
\Delta N_{i, *}^{\mathrm{M} 35}\left(I_{\mathrm{c}}\right)=\left\{0.2^{2} \times\left[\left(N_{i+1, *}\left(I_{\mathrm{c}}\right)-N_{i, *}\left(I_{\mathrm{c}}\right)\right)^{2}+\left(N_{i, *}\left(I_{\mathrm{c}}\right)-N_{i-1, *}\left(I_{\mathrm{c}}\right)\right)^{2}\right]+\left(\Delta N_{\text {field }}\right)^{2}\right\}^{0.5}
$$

The values can be found in column \#5 of Table 3 .

\subsection{The Mass Function}

\subsubsection{Estimating the stellar mass}

We estimated the masses using the $\mathrm{I}_{\mathrm{c}}$ magnitudes. We used several isochrones from different groups, namely D'Antona \& Mazzitelli (1994), D'Antona \& Mazzitelli (1997), Baraffe et al. (1998) and Siess et al. (2000). In the first case, luminosities were converted into bolometric magnitudes and these into $I_{c}$ magnitudes via bolometric corrections of Monet et al. (1992) and the distance modulus of the cluster. Baraffe et al. (1998) and Siess et al. (2000) provide colors and magnitudes. Note that the mass range of the evolutionary tracks is different for each group and that in the last cases they do not cover the whole range of this study.

Figure 7a displays a comparison between the Mass Functions computed with 100 Myr isochrones from each model. From top to bottom, D'Antona \& Mazzitelli (1994) -with the magnitude-mass relation quoted above-, D'Antona \& Mazzitelli (1994) -where we estimated I magnitudes from luminosities based on Siess et al. (2000) data-, D’Antona \& Mazzitelli (1997), Baraffe et al. (1998) and Siess et al. (2000). For each MF, we have fitted a Salpeter law $\psi(\mathrm{M})=\mathrm{k} \times \mathrm{M}^{-\alpha}$, in the mass range $1-0.2 \mathrm{M}_{\odot}$. The fits are shown as solid lines. The power law indices are, respectively, $\alpha=0.98$, 1.10, 1.13, 0.94 and 1.14. For clarity, we have added shifts of 0.4 dex to the MFs compared to the previous one above it. As the figure clearly shows, the general trend is very similar in each case, independent of the model. Below $0.2 \mathrm{M}_{\odot}$ there is a substantial decrease in the number of objects. Note that our completeness limit is below this value, about $0.1-0.08 \mathrm{M}_{\odot}$, depending on the model. Therefore, this structure in the MF indicates that the efficiency of the fragmentation process of the original cloud of the cluster was low below $0.2 \mathrm{M}_{\odot}$, producing a smaller number of objects compare 
with stars more massive than $0.2 \mathrm{M}_{\odot}$. Another possibility is that a fraction of original low mass objects have been expelled during the cluster lifetime (i.e., mass segregation has occurred).

\subsubsection{The effect of the age on the Mass Function.}

As stated before, there is a considerable disagreement on the age of M35. Initial estimates were close to 70-100 Myr, the age of the Pleiades (Vidal 1973) and they were based on photographic and photoelectric photometry. More recent CCD photometry (Sung \& Bessell 1999, von Hippel et al. 2000) indicates that the cluster is somewhat older than the Pleiades, up to 200 Myr. Our own estimate, based on rotational velocity and lithium abundance of bona fide members of the cluster, is 175 Myr. We have tried to avoid this controversial issue by deriving the Mass Function of the cluster with isochrones of different ages. Figure $7 \mathrm{~b}$ shows the results. Isochrones corresponding to ages of 100, 125, 160 and 200 Myr and models from Baraffe et al. (1998) - dashed lines- and Siess et al. (2000) - solid lines- were used to derive the MFs. Arbitrary shifts of 0.3 and 0.5 dex were used to display different MFs. Again, the results are very similar in all cases. The position of the maximum depends slightly on the age of the isochrone and can be moved from $0.2 \mathrm{M}_{\odot}-100 \mathrm{Myr}-$ to $0.25 \mathrm{M}_{\odot}-200 \mathrm{Myr}^{-}$. In general, the effect is a shift of the Mass Function to the left side of the diagram, but keeping the shape of the MF unchanged. Therefore, within the uncertainty in age, we can ignore the effect of age on the MF.

\subsubsection{The complete Mass Function for M35}

A complete Mass Function of the open cluster M35 is displayed in Figure 8. In panels a and b, M35 data are shown as crosses (photometry from Sung \& Bessell 1999, corrected by mass segregation), solid circles $\left(\mathrm{VI}_{c}\right.$ data from KPNO) and open circles $\left(\mathrm{RI}_{c}\right.$ data from $\left.\mathrm{CFHT}\right)$. Panel a also includes a Salpeter law $\psi(\mathrm{M})=\mathrm{k} \times \mathrm{M}^{-\alpha} \quad(\alpha=2.35$, note that only stars more massive than 0.6 $\mathrm{M}_{\odot}$ were used for the fit), whereas panels b and c show the M35 Mass Function in a log-log version. For comparison purposes, we display on this last two figures data from the Pleiades open cluster, as a dashed lines (Figure 8b) or thin solid lines (Figure 8c). Error bars are included for our M35 data.

Figure 8a clearly shows that a Salpeter law does not fit the whole Mass Function of the cluster. We have fitted three different power laws, yielding $\alpha=+2.59 \pm 0.04$ for the mass range $6-0.8 \mathrm{M}_{\odot}$, $\alpha=+0.81 \pm 0.02$ for $0.8-0.2 \mathrm{M}_{\odot}, \alpha=-0.88 \pm 0.12$ for $0.2-0.08 \mathrm{M}_{\odot}$ (Figure $8 \mathrm{~b}$ ). Note that the mass limits are different to those used in previous subsections and in Figure 7a. For the upper part of the main sequence of M35, Leonard \& Merritt (1989) obtained $\alpha=+2.7 \pm 0.4\left(6-1 \mathrm{M}_{\odot}\right)$. In the case of M35 stars slightly cooler, Sung \& Bessell (1999) derived $\alpha=+2.1 \pm 0.3\left(4.4-0.7 \mathrm{M}_{\odot}\right)$. Our M35 Mass Function can be compared with the MF for the Pleiades, computed for mass below $\sim 0.5 \mathrm{M}_{\odot}$, by Martín et al. (1998) $-\alpha=+1.00 \pm 0.15,0.4-0.04 \mathrm{M}_{\odot^{-}}$, Bouvier et al. (1998) $-\alpha=+0.6,0.4-0.06 \mathrm{M}_{\odot^{-}}$, 
Hambly et al. (1999) $-\alpha=+0.7,0.6-0.06 \mathrm{M}_{\odot}^{-}$, Hodgkin \& Jameson $(2000)-\alpha=+0.8,0.50-0.04$ $\mathrm{M}_{\odot^{-}}$, and Martín et al. (2000) $-\alpha=+0.53,0.4-0.06 \mathrm{M}_{\odot^{-}}$. All these MFs, arbitrarily scaled, are shown in Figure 8b as solid and dashed lines. In all cases, the power law indices are quite similar, when we compare similar mass ranges. Note that our statistics are much better due to the richness of the M35 cluster compared with the Pleiades.

A comparison between a complete Mass Functions of M35 (this work) and the Pleiades (Hambly et al. 1999) can be found in Figure 8c. Solid circles correspond to M35 data, whereas solid triangles represent the Pleiades. Note the remarkable similarity between both clusters, despite the different age, metallicity and total mass (see next subsection). We interpret the difference in the mass range 0.3-0.08 $\mathrm{M}_{\odot}$ essentially as arising from the different binning between our data and the dataset by Hambly et al. (1999). Since we have a large number of candidate members at the bottom of the main sequence, we have been able to discriminate smaller bins close to the substellar limit, i.e., we have four points in the area around $0.1 \mathrm{M}_{\odot}$, where the Pleiades data presents an apparent gap, due to its wide bins. Therefore, we are able to distinguish more structure, and the real decrease of the MF in this mass range. However, we cannot rule out the possibility of a real difference in the MFs, even a mass segregation in the case of M35 for very low mass objects. For instance, BD searches in the Hyades (600-800 Myr) have not produced any positive identification (Gizis et al. 1999). Figure 8c also includes data from Gould et al. (1997), corresponding to field stars. This dataset presents a flat $\mathrm{MF}$ in the mass range $0.5-0.08 \mathrm{M}_{\odot}$, with a step and narrow decrease at 0.1 $\mathrm{M}_{\odot}$. As shown by other authors in the Pleiades and in the very young cluster (1-5Myr) associated to sigma Orionis (Béjar et al. 2000), the MF increases again below the substellar limit.

In summary, it seems that the Mass Function presents four different types of behavior: From the top of the main sequence down to $\sim 0.8 \mathrm{M}_{\odot}$, the MF is very step, with a power index $\alpha \sim+2.6$ (where $\psi(\mathrm{M})=\mathrm{k} \times \mathrm{M}^{-\alpha}$ ). In the mass range $0.8-0.2 \mathrm{M}_{\odot}$, the $\mathrm{MF}$ is shallower, with a index close to $\alpha \sim+0.9$. Between this point and the substellar limit, a real decreases in the M35 Mass Function takes place. The index can be evaluated as $\alpha=-0.8$. Finally, as shown by Béjar et al. (2000) in the whole substellar domain, the tendency is inverted and a new increment in the Mass Function appears, with $\alpha=+0.8$. These different power law indices suggest that several mechanism (at least three) are acting during the fragmentation and collapse of the original cloud, competing with each other.

\subsubsection{The total mass of the cluster}

The total dynamical mass of M35 has been estimated as 1600-3200 $\mathrm{M}_{\odot}$ (Leonard \& Merritt 1989). Using mass function arguments, Sung \& Bessell (1999) computed a total mass of $1660 \mathrm{M}_{\odot}$. The average mass of a star is $0.4 \mathrm{M}_{\odot}$, which means M35 should contain from 4000 to 8000 members. Prior to our study, about 1500 candidate members of M35 were known. We have proposed another $\sim 1700$, which have a contamination rate close to $30 \%$. In total, about 2700 members of M35 have

been identified so far. This fact seems to indicate that the total mass of the cluster is closer to the 
$1600 \mathrm{M}_{\odot}$ estimate than to the $3200 \mathrm{M}_{\odot}$. In fact, our own estimates are in excellent agreement with this rough estimate.

We have used the complete dataset displayed in Figure 3c to derive the total mass of the cluster. This sample includes our new candidate members as well as all photometric members discovered previously. Masses were derived using a 100 Myr isochrone (D’Antona \& Mazzitelli 1994).

Figure $9 \mathrm{a}, 9 \mathrm{~b}$ and $9 \mathrm{c}$ represent the cumulative total mass distribution against the $\mathrm{M}\left(\mathrm{I}_{\mathrm{c}}\right)$ absolute magnitude, $(\mathrm{V}-\mathrm{I})_{\mathrm{c}, 0}$ unreddened color index and the individual mass, respectively. In the case of Figures 9a and c, we derived masses from the I magnitudes, whereas in the case of Figure 9b, we used the color. Essentially, results are very similar. The vertical long-dashed straight segment represents the location of the substellar limit (Figure 9b and c). The other three curves represent different calculations of the cumulative total mass of the cluster: The dotted line correspond to all probable members and candidate members. Therefore, it is affected by the field star pollution. The thick solid line represents the optimal estimate, where we included all probable members (using proper motions and radial velocities) and candidate members (our candidate members and previously known candidate members without known proper motions), but removing the spurious members $(\sim 30 \%)$. Finally, the short-dashed solid line corresponds to the probable members and candidate members once the field contamination has been removed. In this last case, we have not included candidate members from previous surveys (i.e., members with no additional information regarding the proper motion and/or the radial velocity) and the final value represents the minimum total mass of the cluster. The location of this value is indicate with a horizontal segment in Figure $9 \mathrm{c}$.

Figure 9c clearly shows that the main contribution to the total mass of the cluster is produced by stars in the mass range $3-0.5 \mathrm{M}_{\odot}$, with a inflection point at $0.8 \mathrm{M}_{\odot}$. The $0.8 \mathrm{M}_{\odot}$ point is also the location of another inflection point in the cluster MF, where the spectral index of the power law changes from $\alpha=+2.59$ to $\alpha=+0.81$.

The total mass within the area covered by this survey is $\sim 2100,1600$ and $1100 \mathrm{M}_{\odot}$, for each case described above, where the value in the middle $\left(1600 \mathrm{M}_{\odot}\right)$ can be considered as a good estimate of the total mass of the open cluster M35 in the considered region. As a comparison, the total mass of the Pleiades has been estimated as 1000-1200 $\mathrm{M}_{\odot}$ (Meusinger et al. 1996) or $735 \mathrm{M}_{\odot}$, (Pinfield et al. 1998), and the total mass of Praesepe is about $626 \mathrm{M}_{\odot}$ (Holland et al. 2000). Note that some remaining M35 members should be located in the corona of the cluster, where deep photometric and proper motion surveys have not been carried out. Therefore, the total mass of M35 should be considerably larger than the mass of the Pleiades. Pinfield et al. (1998) have estimated that the average mass of a Pleiades star is $<$ mass $>=0.616 \mathrm{M}_{\odot}$. In the case of M35, the average mass is $<$ mass $>=0.602 \mathrm{M}_{\odot}$, very similar despite the difference in the number of stars in each cluster (725 against 2713 , respectively). 


\section{Summary and Conclusions}

We have obtained deep $\mathrm{V}(\mathrm{RI})_{\mathrm{c}}$ photometry of the open cluster M35, reaching much fainter stars than the previous surveys. The comparison of the location of the stars in color-magnitude and color-color diagrams allowed us to establish the preliminary membership of the detected stars. We have also estimated the contamination by field stars. We have analyzed this accurate, deep photometry of M35. Our survey has shown that in the studied color range there is a weak mass segregation, in opposition to the stronger segregation present for more massive stars. These data have allowed us to establish the Luminosity Funtion and Mass Function of the low and very low mass components of the clusters. The LF presents a peak at $\mathrm{I}_{\mathrm{C}}=19$ magnitudes, corresponding to a mass of $\sim 0.3 \mathrm{M}_{\odot}$. This result is very similar to the Pleiades' LF. By merging our own dataset with data from previous studies of the cluster, which correspond to more massive stars, we have been able to obtain a complete mass function for the stellar component of the cluster, covering the mass range $6.0 \leq$ Mass $\leq 0.08 \mathrm{M}_{\odot}$. When expressed in a log-log form, the MF increases monotonically in the 6-0.8 $\mathrm{M}_{\odot}$ domain with a power index of $\alpha=+2.59$. After this point, the power index is reduced significantly, to a value of $\alpha=+0.81$. Below this point, the behavior changes and the power law index become negative $(\alpha=-0.8)$. Finally, we have estimated the total mass of the cluster in the area were our data were collected. After taking into account the pollution by field stars, the total mass contained in the central part of the cluster is $\sim 1600 \mathrm{M}_{\odot}$. It would be very interesting to collect deeper photometry of the cluster, reaching well below the substellar limit, to verify whether the M35 MF behaves as the Pleiades and sigma Orionis Cluster or if a significant fraction of the M35 BDs have already been dissipated from the cluster.

DBN thanks the "Instituto Astrofísico de Canarias" and "Ministerio de Educación y Cultura" (Spain), and the "Deutsche Forschungsgemeinschaft" (Germany) for their fellowship. JRS acknowledges support from NASA Grant NAGW-2698. This work has been partially suported by Spanish "Plan Nacional del Espacio", under grant ESP98-1339-CO2. The comparison with previously published data has been much easier due to the work done by J.-C. Mermilliod, via his WEBDA database. We greatly appreciate this contribution to the astronomical community. We thank the referee, M.S. Bessell, for his multiple and useful comments.

\section{REFERENCES}

Baraffe, I., Chabrier, G., Allard, F., Hauschildt, P.H., 1998, A\&A 337, 403

Baraffe, I., 1998, priv. comm.

Barrado, D., Byrne, B.P., 1995, A\&A SS 111, 275

Barrado y Navascués, D., Stauffer, J.R., Randich, S., 1997, Mem.S.A.It. 68, 985

Barrado y Navascués, D., Stauffer, J.R., Randich, S., 1998a, ApJ 506, 347 
Barrado y Navascués, D., Stauffer, J.R., Bouvier, J., Martín, E.L., 1998b, Ap\&SS 263, 303

Barrado y Navascués, D., Stauffer, J.R., Song, I., Caillault, J-P., 1999, ApJ Letters 520, L123

Barrado y Navascués, D., Deliyannis, C.P., Stauffer, J.R., 2000a, in "Clusters and Associations: Convection, Rotation, and Dynamos". R. Pallavicini, G. Micela and S. Sciortino (eds.) ASP Conf. Series 198, 265

Barrado y Navascués, D., Deliyannis, C.P., Stauffer, J.R., 2000b, ApJ, submitted

Barrado y Navascués D., Stauffer J.R., Briceño C., Patten B.M., Hambly N., Adams, J. 2000c, ApJ, submitted

Béjar V., Martín E.L., Zapatero Osorio M.R., Rebolo, R., Barrado y Navascués D., Bailer-Jones, C., Mundt, R., Baraffe I., Chabrier G., Allard, F., 2000, ApJ Letters, submitted

Bessel, M.S., 1991, AJ 101, 662

Bouvier J., Stauffer R., Martín E.L., Barrado y Navascués D., Wallace B., Béjar V., 1998, A\&A, 336,490

Cousins A.W.J., 1978, Mon.Not.Astron.Soc.S.Afr. 37, 62

Cudworth, K.M., 1971, AJ 76, 475

Cuffey J., 1938, Ann. Harvard College Obs. 106, 39

D’Antona, F., \& Mazzitelli, I., 1994, ApJS 90, 467

D’Antona, F., \& Mazzitelli, I., 1997, Mem.S.A.It. 68, 807

Giclas, H.L., Burnham, R., Thomas, N.G., 1971, Lowell Proper Motion Survey.

Gizis J.E., Reid I.N., Monet D. G., 1999, AJ 118, 997

Gliese, W., 1969, Weroeff. Astron. Rechen-Inst. 22, 1

Gliese, W., Jahreiss, H., 1979, A\&A SS 38, 423

Gould A., Bahcall J.N., Flynn C., 1997, ApJ 482, 913

Hambly N.C., Hodgkin S.T., Cossburn M.R., Jameson R.F., 1999, MNRAS 303, 835

Hoag, A.A., Johnson, H.L., Iriarte, B., Mitchell, R.I., Hallam, K.L., Sharpless, S., 1961, Publ. U.S. Naval Obs. 17, 345

Hodgkin S.T., Jameson R.F., 2000, in "Clusters and Associations: Convection, Rotation, and Dynamos". R. Pallavicini, G. Micela and S. Sciortino (eds.) ASP Conf. Series 198, 59 
Holland K., Jameson R.F., Hodgkin S.T., Davies M., 2000, in "Clusters and Associations: Convection, Rotation, and Dynamos". R. Pallavicini, G. Micela and S. Sciortino (eds.) ASP Conf. Series 198, 191

Landolt A.U., 1992, AJ 104, 340

Leggett, S.K. 1992, ApJS 82, 351

Leonard, P.J.T, Merritt, D., 1989, ApJ 339, 195

Luhman, K.L., Rieke, G.H., Lada, C.J., Lada, E.A., 1998, ApJ 503, 347

Luyten, W.J., 1972, Proper Motion Survey with the 48-inch Telescope, Univ. Minnesota, 29, 1

Martín E.L., Zapatero Osorio, M.R., Rebolo, R., 1998, in "Brown Dwarfs and Extrasolar Planets", R. Rebolo, E.L. Martín, M.R., Zapatero Osorio (eds.), APS Conf. Series 134, 507.

Martín E.L., Bradner W., Bouvier J., Luhman K.L., Stauffer J.R., Basri G., Zapatero Osorio M.R., Barrado y Navascués D., 2000, ApJ, in press

McNamara, B., Sekiguchi, K., 1986a, AJ 91, 557

McNamara, B., Sekiguchi, K., 1986b, ApJ 310, 613

Mermilliod J.-C., 1981 A\&A 97, 235

Mermilliod J.-C., 1996 in "The origins, evolution, and destinies of binary stars in clusters", E.F. Milone and J.-C. Mermilliod (eds.), ASP Conf. Series 90, 475

Meusinger H., Schilbach E., Souchay J., 1996, A\&A 312, 833

Meynet G., Mermilliod J.-C., \& Maeder A., 1993, A\&ASS 98, 477

Monet D.G., Dahn C.C., Vrba F.J., Harris, H.C., Pier J.R., Luginbuhl C.B., Ables H.D., 1992, AJ 103,638

Pinfield D.J., Jameson R.F., Hodgkin S.T., 1998, MNRAS 299, 955

Randich, S., Schmitt, J.H.M.M., 1995, A\&A 298, 134

Sarrazine, A. R., Steinhauer, A. J. B., Deliyannis, C. P., Sarajedini, A., Bailyn, C. D., KozhurinaPlatais, V., von Hippel, T., \& Platais, I., 2000, AAS, 32, \#742.

Siess L., Dufour E., Forestini M., 2000, A\&A 358, 593

Stauffer, J.R., Schultz, Greg, Kirkpatrick, D., 1998, ApL Letters 499, L199

Stauffer J.R., Barrado y Navascués D., Bouvier J., Morrison H.L., Hardig P., Luhman K., Stanke T., McCaughrean M., Terndrup D.M., Allen L., \& Assouad P. 1999, ApJ 527, 219 
Stauffer J.R., Barrado y Navascués D., 2000, in "11th Cambridge Workshop on Cool Stars, Stellar Systems and the Sun", R. García López, R. Rebolo, M.R. Zapatero-Osorio (eds), ASP Conf. Series, in press.

Sung H., Lee S-W., 1992, JKAS 25, 91

Sung H., Bessell M.S. 1999, MNRAS 306, 361

Vidal, N.V, 1973, A\&A SS 11, 93

von Hippel, T., Kozhurina-Platais, V., Platais, I., Demarque, P., Sarajedini, A., 2000, in "Clusters and Associations: Convection, Rotation, and Dynamos". R. Pallavicini, G. Micela and S. Sciortino (eds.) ASP Conf. Series 198, 75

Zapatero Osorio, M.R., 1997, PhD. Universidad de La Laguna, Spain 
Fig. 1.- a KPNO bright data: V magnitude against (V-I) color, shallow exposure. Solid circles indicate the position of bona fide M35 members, based on radial velocity data (Barrado y Navascués et al. 2000a,b). The dashed and solid lines represent the completeness and detection limits, respectively. b KPNO bright data: V magnitude against (V-I) color, deep exposure. Symbols as Figure 1a. c CFHT deep data: $I_{c}$ magnitude against $(R-I)_{c}$ color index. The dashed line represent the completeness limit.

Fig. 2.- Color-color magnitude diagram for M35. Solid circles represent the the M35 candidates selected as photometric members, whereas the crosses indicate the position of the rejected candidates (see text). As a comparison, we show IC2391 probable members (asterisks) from Barrado y Navascués et al. (2000c). The solid line represents the relation derived using Leggett (1992) data, whereas the dashed line corresponds to the relation for M dwarfs published by Bessell (1991).

Fig. 3.- a Final color-magnitude diagram for M35, KPNO data. Radial velocity members appear as solid circles (from Barrado y Navascués et al. 2000a,b), whereas other candidate members are displayed as open circles. Rejected candidate members, based on Figure 2, appear as crosses. Members from Sung \& Bessell (1999) are shown as asterisk symbols. b Final M35 color-magnitude diagram, CFHT data. Solid circle represent stars with $\mathrm{V}(\mathrm{RI})_{\mathrm{c}}$ data in agreement with membership, whereas open circles represent candidate members which only have RI photometry. c A Complete color-magnitude diagram for the open cluster M35. The diagram includes all our candidate members and the bona fide members by Sung \& Bessell (1999). Note that for the faint end (V-I) $\geq 2.0$, most of the color indices were derived from the (R-I), using our empirical MS.

Fig. 4.- Estimation of the pollution by field stars fitting Gaussian curves. We show one of the cuts along the $\mathrm{I}_{c}$ axis in the color-magnitude diagram. Three Gaussian curves were fitted as described in the text. The dotted line represents the fitted field star contribution.

Fig. 5.- Mass segregation in the cluster. Open circles, solid triangles and open triangles represent data from Sung \& Bessell (1999), KPNO and CFHT, respectively.

Fig. 6.- Luminosity Function. Solid and open circles represent data from KPNO data and CFHT data, respectively.

Fig. 7.- a Comparison of several M35 MFs derived with different models. The power law index for each Mass Function is shown. In all cases, 100 Myr isochrones were used. For clarity, we have added arbitrary shifts of 0.4 dex, respect the curve inmediately above. b Comparison of several M35 MFs derived with different isochrones and models. Models from Siess et al. (2000) appear as solid lines and circles, whereas isochrones from Baraffe et al. (1998) are plotted as dashed lines and open circles. For clarity, we have added arbitrary shifts of 0.3 and 0.5 dex.

Fig. 8. - a Complete Mass Function of M35. Crosses, solid and open circles represent data from Leonard \& Merritt (1989), KPNO data and CFHT data, respectively. The solid line is a MF with a Salpeter index $(\alpha=2.35)$, for stars more massive than $0.6 \mathrm{M}_{\odot}$. b Complete Mass Function of 
M35 in logarithmic form (thick solid line). The results from Leonard \& Merritt (1989) and Sung \& Bessell (1999) are displayed as thin solid lines. Different Pleiades MFs, scaled with arbitrary shifs, are shown as dashed lines. From top to bottom: Martín et al. (1998), Hodgkin \& Jameson (2000), Hambly et al. (1999), Bouvier et al. (1998) and Martín et al. (2000). The spectral power indices of the MF are indicated inside the parenthesis. c Complete Mass Function of M35 in logarithmic form (solid circles). The best fit is shown as a thick solid line. As comparison, the complete Mass Funtion of the Pleiades is shown as solid triangles (Hambly et al. 1999), with an arbitrary shift of 0.4 dex. The fits and the power law indices within their range are also plotted in the Pleiades case. Data from Gould et al. (1997), corresponding to field stars, are included as crosses.

Fig. 9.- a Cumulative total mass of the cluster versus the apparent $I_{c}$ magnitude (top) or the absolute $\mathrm{M}\left(\mathrm{I}_{\mathrm{c}}\right)$ magnitude (bottom). Different lines represent several estimates of the total mass of the cluster (see text). Stellar masses were derived from the $I_{c}$ magnitudes. $b$ Cumulative total mass of the cluster versus the dereddened $(\mathrm{V}-\mathrm{I})_{\mathrm{c}}$ color index. Different lines represent several estimates of the total mass of the cluster (see text). The vertical long-dashed line represent the location of the substellar border at $\sim 0.075 \mathrm{M}_{\odot}$ for a $100 \mathrm{Myr}$ old object. Stellar masses were derived from the $(\mathrm{V}-\mathrm{I})_{\mathrm{c}}$ color indices. c Cumulative total mass distribution of the cluster versus the individual mass. The minimum total mass for this last case is indicated with a horizontal segment. Symbols as in Figure 8b. Stellar masses were derived from the $I_{c}$ magnitudes. 
Table 1: Empirical M35 main sequence. We used $(\mathrm{m}-\mathrm{M})_{0}=9.60, \mathrm{E}(\mathrm{B}-\mathrm{V})=0.255, \mathrm{E}(\mathrm{V}-\mathrm{I})_{\mathrm{c}}=0.321$ and $\mathrm{E}(\mathrm{R}-\mathrm{I})_{\mathrm{c}}=0.178$, with $\mathrm{R}_{\mathrm{V}}=3.0$.

\begin{tabular}{ccc}
\hline $\mathrm{V}$ & $(\mathrm{V}-\mathrm{I})_{c}$ & $(\mathrm{R}-\mathrm{I})_{c}$ \\
$(1)$ & $(2)$ & $(3)$ \\
\hline 12.544 & 0.410 & - \\
12.919 & 0.519 & - \\
13.391 & 0.640 & 0.317 \\
13.513 & 0.675 & 0.336 \\
13.864 & 0.761 & 0.363 \\
14.083 & 0.801 & 0.378 \\
14.368 & 0.827 & 0.389 \\
14.621 & 0.855 & 0.401 \\
14.922 & 0.901 & 0.419 \\
15.468 & 0.961 & 0.440 \\
15.969 & 1.066 & 0.479 \\
16.228 & 1.115 & 0.505 \\
16.828 & 1.245 & 0.591 \\
17.786 & 1.490 & 0.697 \\
18.676 & 1.806 & 0.916 \\
19.952 & 2.294 & 1.278 \\
20.741 & 2.540 & 1.396 \\
22.419 & 2.986 & 1.695 \\
23.244 & 3.111 & 1.772 \\
24.815 & 3.499 & 1.983 \\
25.458 & 3.654 & 2.068 \\
26.100 & 3.946 & 2.208 \\
26.984 & 4.270 & 2.348 \\
27.477 & 4.603 & 2.478 \\
28.194 & 4.940 & 2.598 \\
\hline & &
\end{tabular}


Table 3: Data for the LM and MF for the low mass and very low mass stars of M35

\begin{tabular}{cccrrrrrc}
\hline $\mathrm{I}_{\mathrm{c}}$ bin & $(\mathrm{V}-\mathrm{I})_{c}$ & $(\mathrm{R}-\mathrm{I})_{c}$ & Contam. & $\mathrm{N}_{i, *}^{\mathrm{M} 35}\left(\mathrm{I}_{\mathrm{c}}\right)$ & Mass & $\Delta$ Mass & $\Delta \mathrm{N} / \Delta \mathrm{M}$ & Dataset \\
$(1)$ & $(2)$ & $(3)$ & $(4)$ & $(5)$ & $(6)$ & $(7)$ & $(8)$ & $(9)$ \\
\hline 13.25 & 0.79 & - & 0.40 & $37.9 \pm 04.4$ & 1.356 & 0.138 & $275 \pm 031$ & kpno \\
13.75 & 0.85 & - & 0.35 & $48.4 \pm 05.3$ & 1.239 & 0.124 & $390 \pm 043$ & kpno \\
14.25 & 0.92 & - & 0.30 & $52.2 \pm 10.0$ & 1.120 & 0.110 & $474 \pm 091$ & kpno \\
14.75 & 1.02 & - & 0.30 & $67.9 \pm 09.1$ & 1.015 & 0.102 & $665 \pm 091$ & kpno \\
15.25 & 1.14 & - & 0.30 & $64.0 \pm 07.0$ & 0.915 & 0.097 & $659 \pm 072$ & kpno \\
15.75 & 1.29 & - & 0.30 & $78.7 \pm 08.4$ & 0.822 & 0.087 & $904 \pm 097$ & kpno \\
16.25 & 1.46 & - & $\sim 0.30$ & $83.6 \pm 08.4$ & 0.742 & 0.078 & $1072 \pm 108$ & kpno \\
16.75 & 1.73 & - & $\sim 0.30$ & $79.7 \pm 09.0$ & 0.657 & 0.092 & $866 \pm 098$ & kpno \\
17.25 & 2.05 & - & $\sim 0.30$ & $100.3 \pm 11.2$ & 0.569 & 0.077 & $1303 \pm 146$ & kpno \\
17.75 & - & 1.25 & $\sim 0.02$ & $115.5 \pm 21.2$ & 0.497 & 0.075 & $1540 \pm 283$ & cfht \\
18.25 & - & 1.35 & $\sim 0.02$ & $138.0 \pm 17.5$ & 0.417 & 0.085 & $1624 \pm 206$ & cfht \\
18.75 & - & 1.55 & $\sim 0.02$ & $187.5 \pm 21.5$ & 0.330 & 0.085 & $2206 \pm 253$ & cfht \\
19.25 & - & 1.65 & 0.07 & $168.9 \pm 17.3$ & 0.252 & 0.069 & $2448 \pm 251$ & cfht \\
19.75 & - & 1.65 & 0.35 & $166.3 \pm 23.9$ & 0.191 & 0.052 & $2819 \pm 460$ & cfht \\
20.25 & - & 1.75 & 0.60 & $80.5 \pm 19.5$ & 0.147 & 0.039 & $2064 \pm 500$ & cfht \\
20.75 & - & 1.85 & 0.60 & $58.5 \pm 09.0$ & 0.112 & 0.031 & $1887 \pm 290$ & cfht \\
21.25 & - & 1.95 & 0.60 & $32.3 \pm 06.9$ & 0.088 & 0.012 & $2691 \pm 575$ & cfht \\
\hline
\end{tabular}




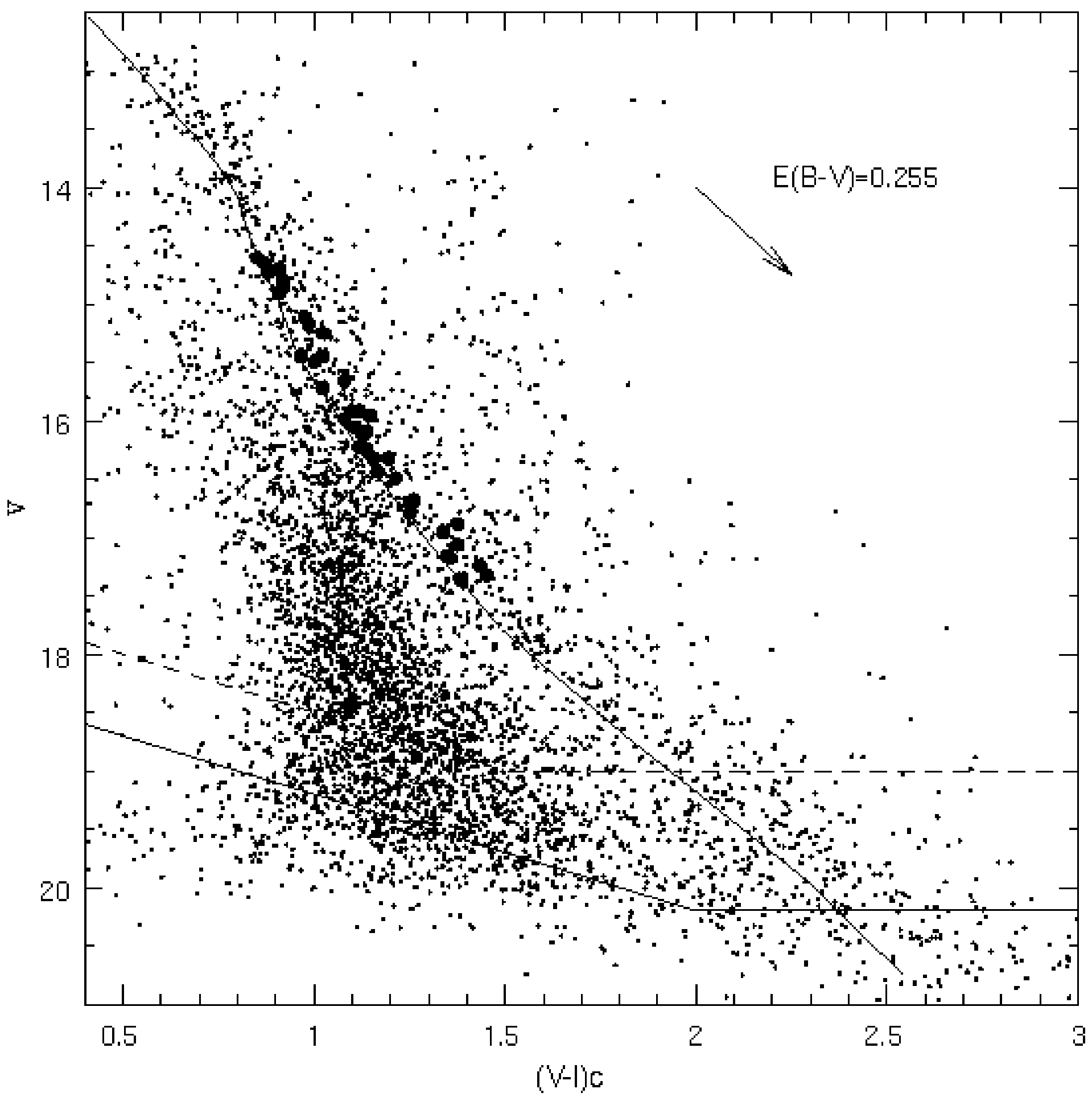




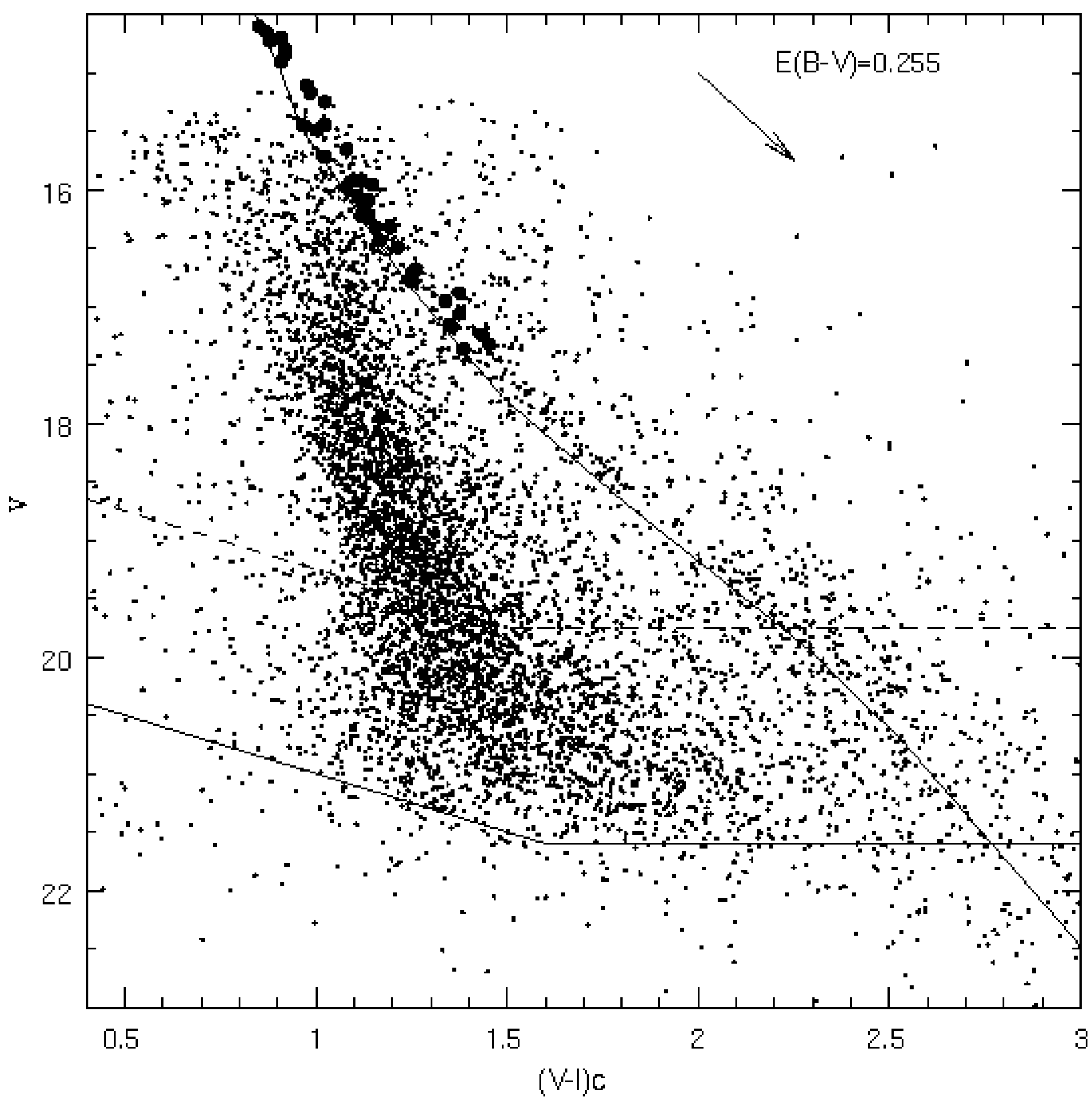




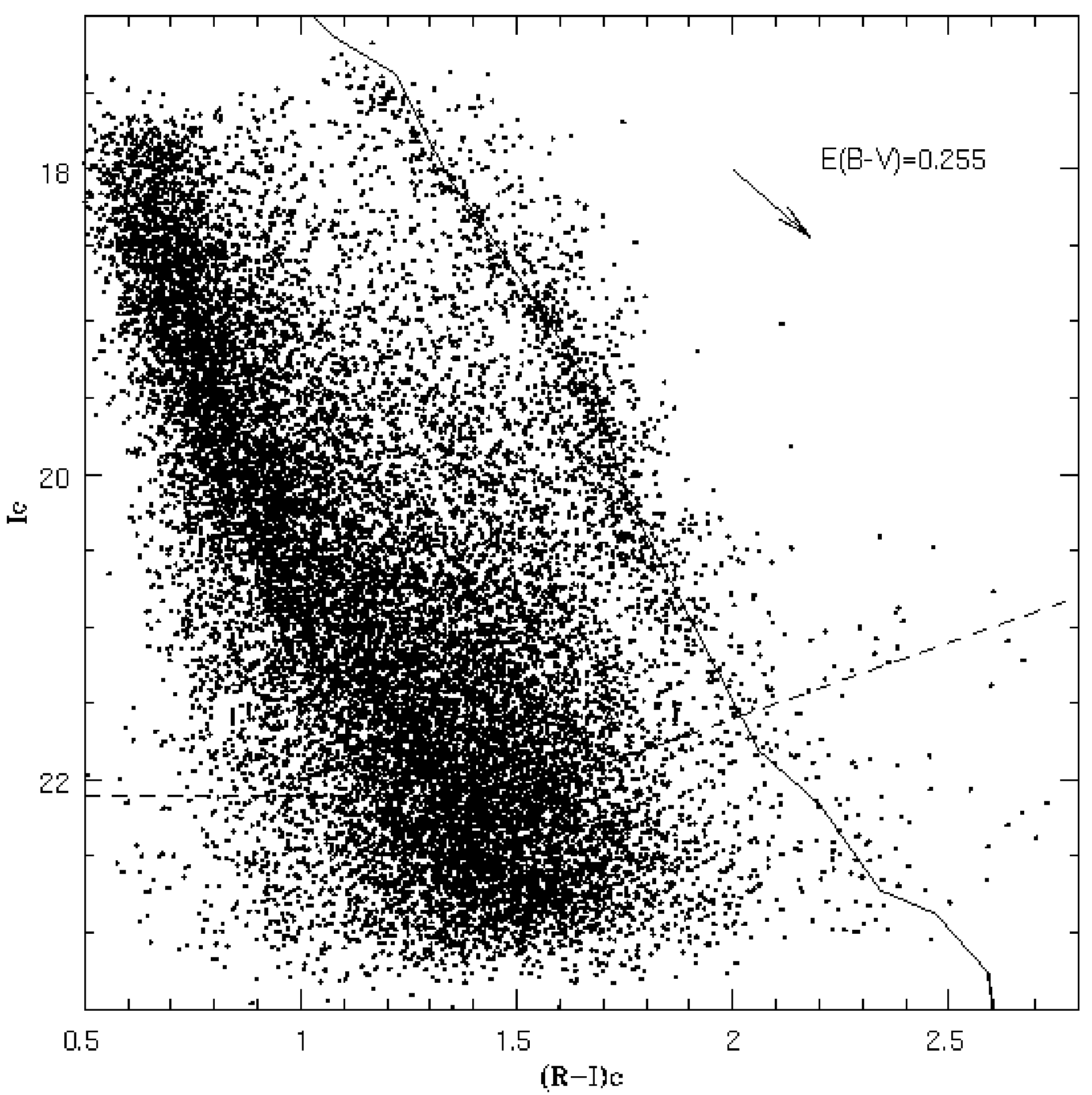




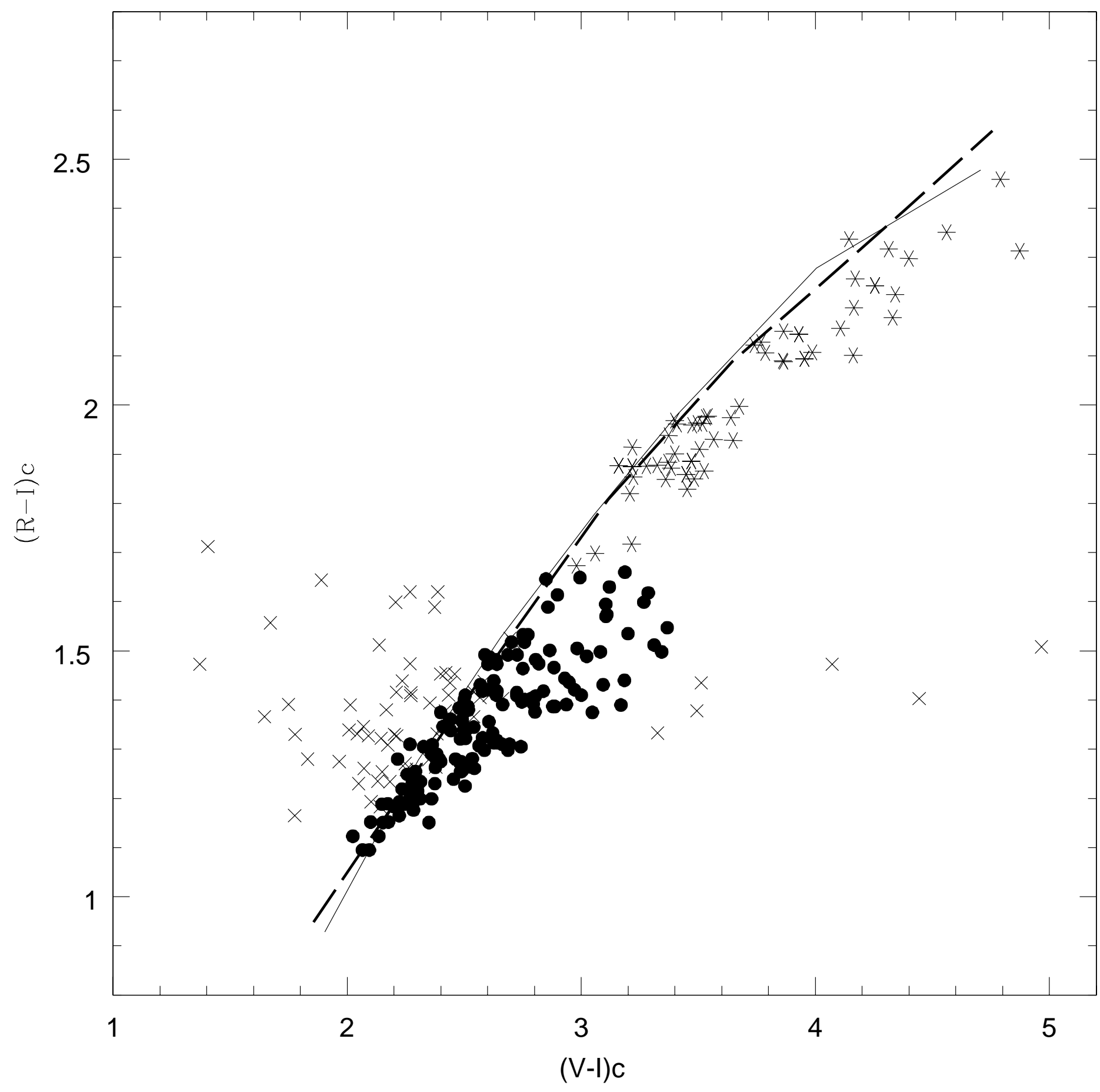




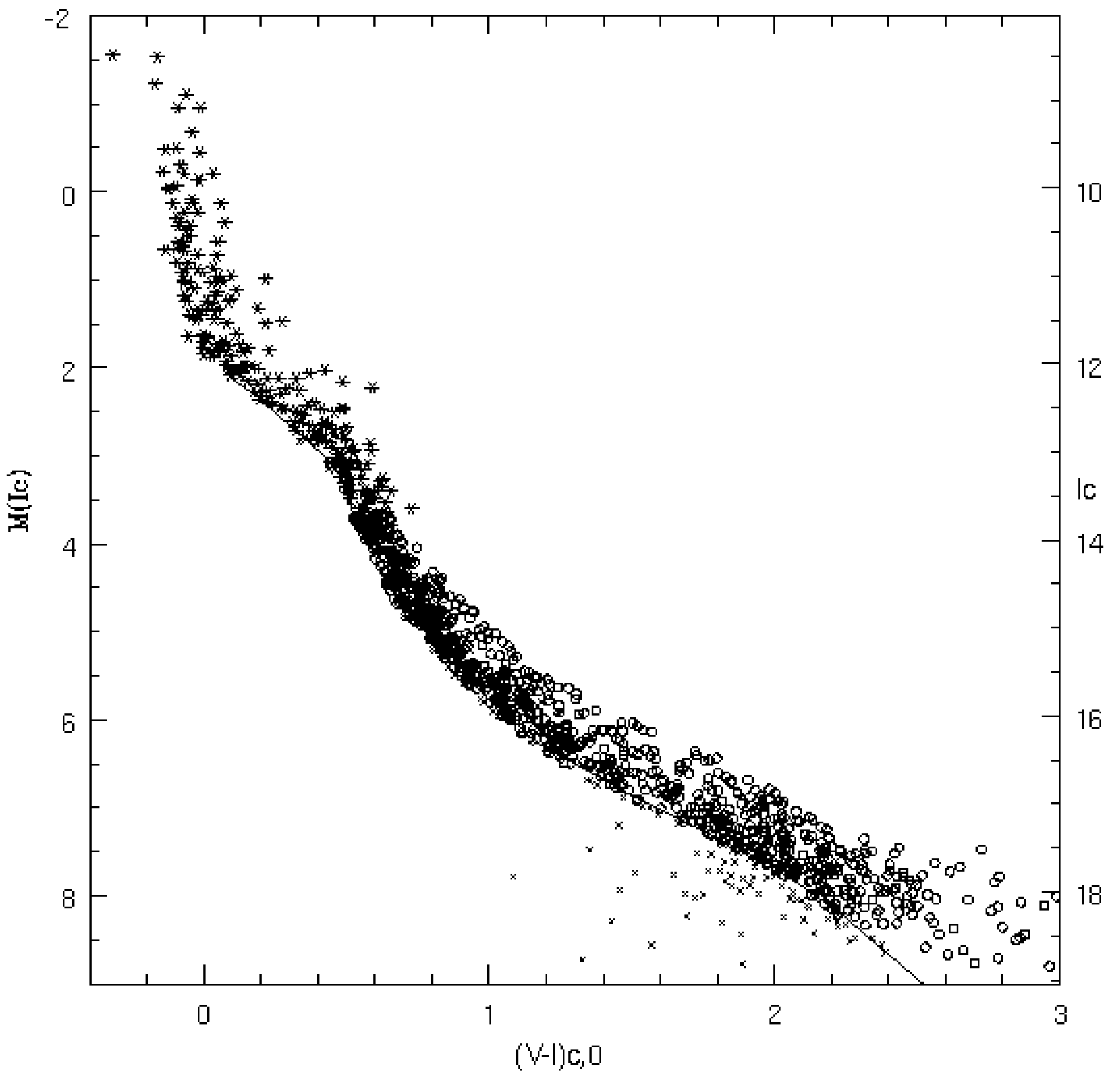




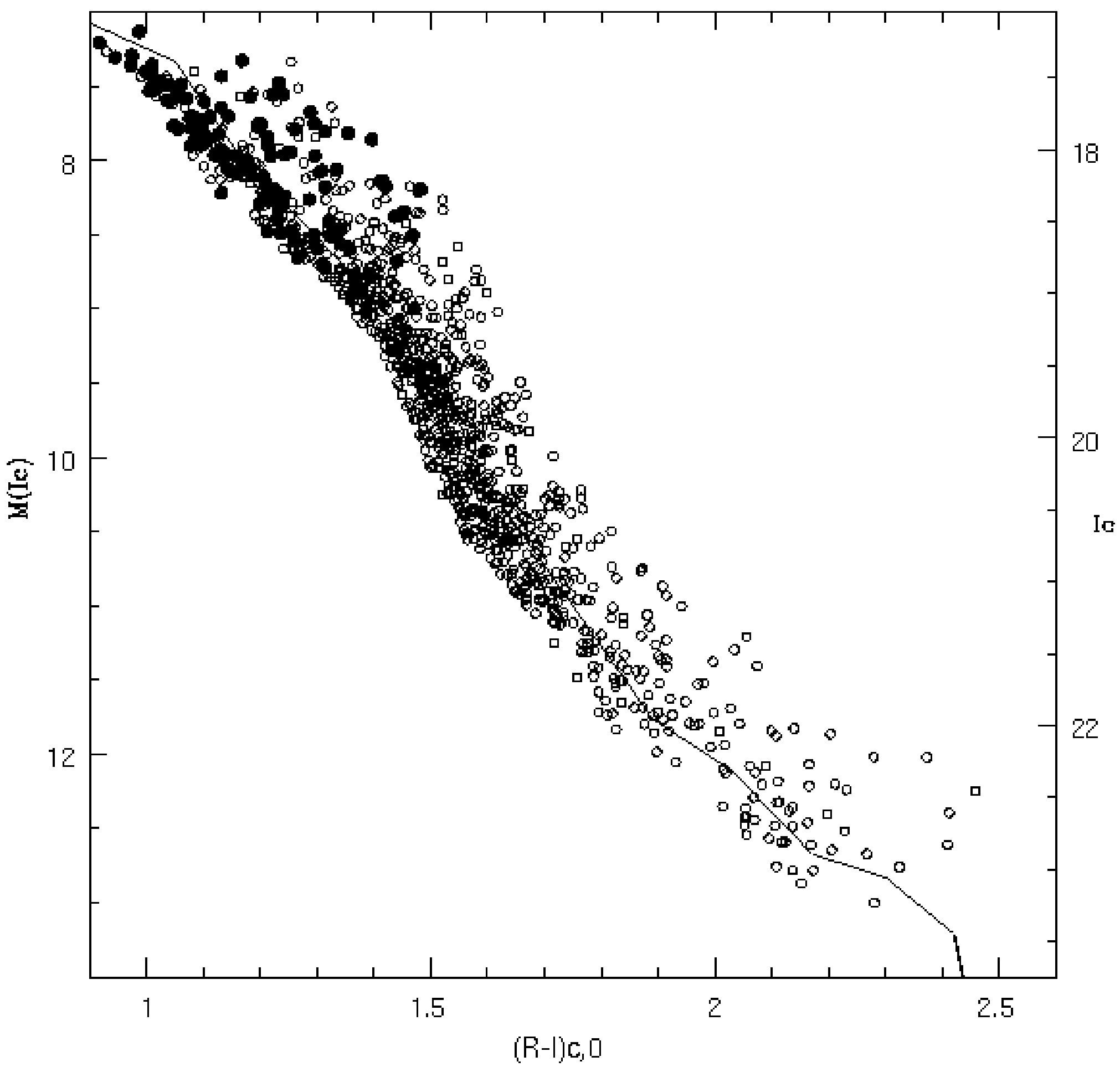




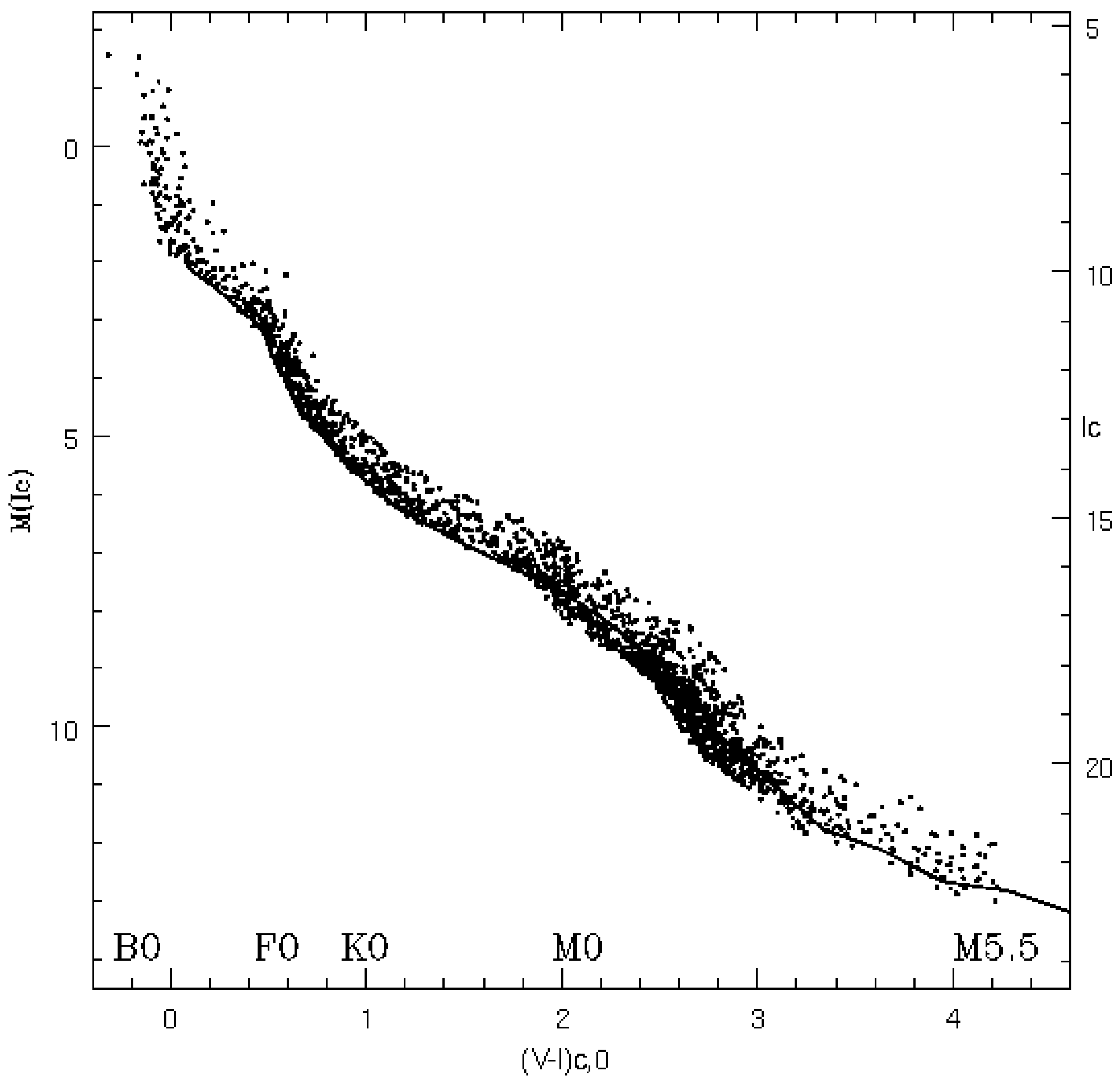




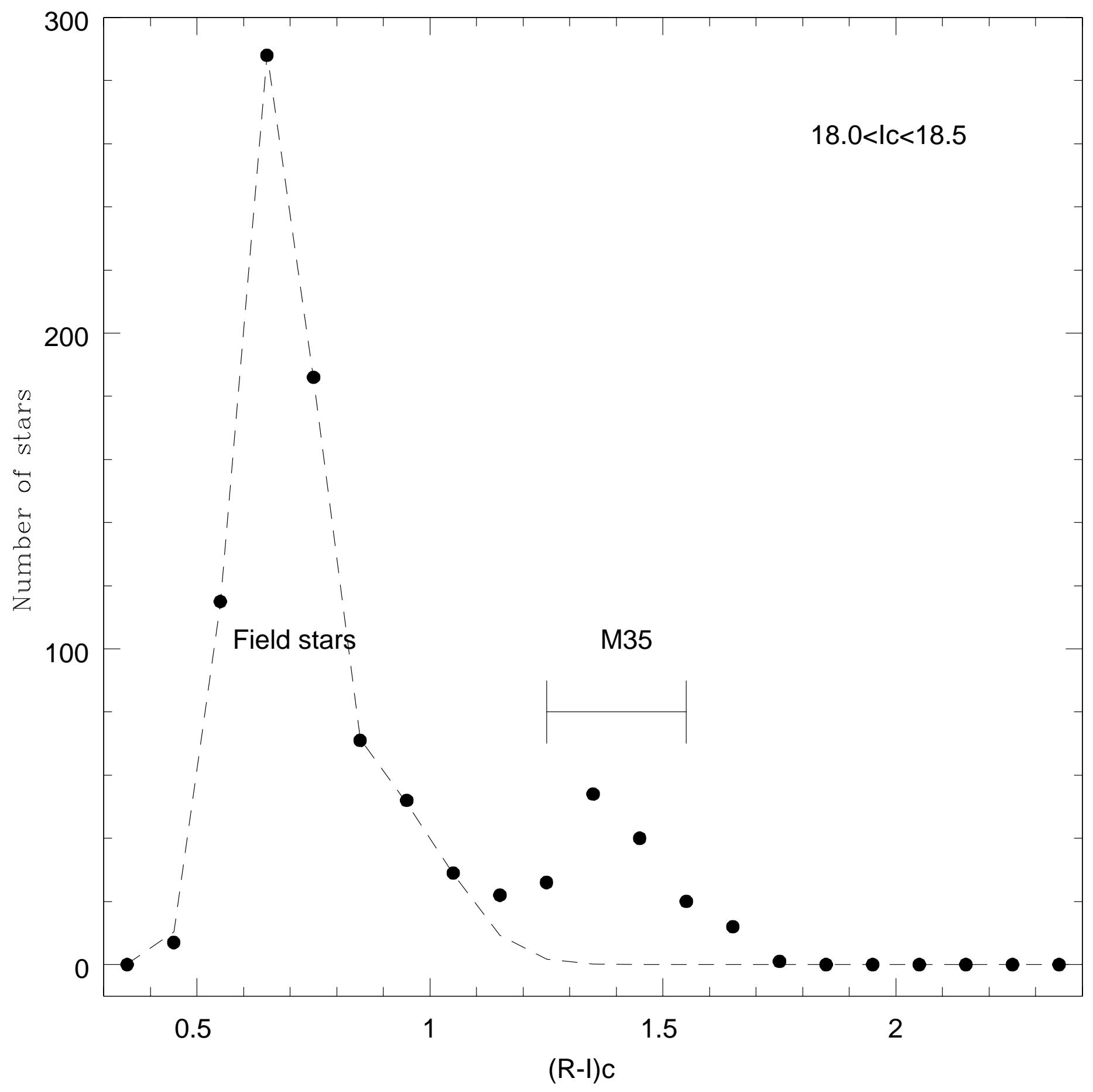









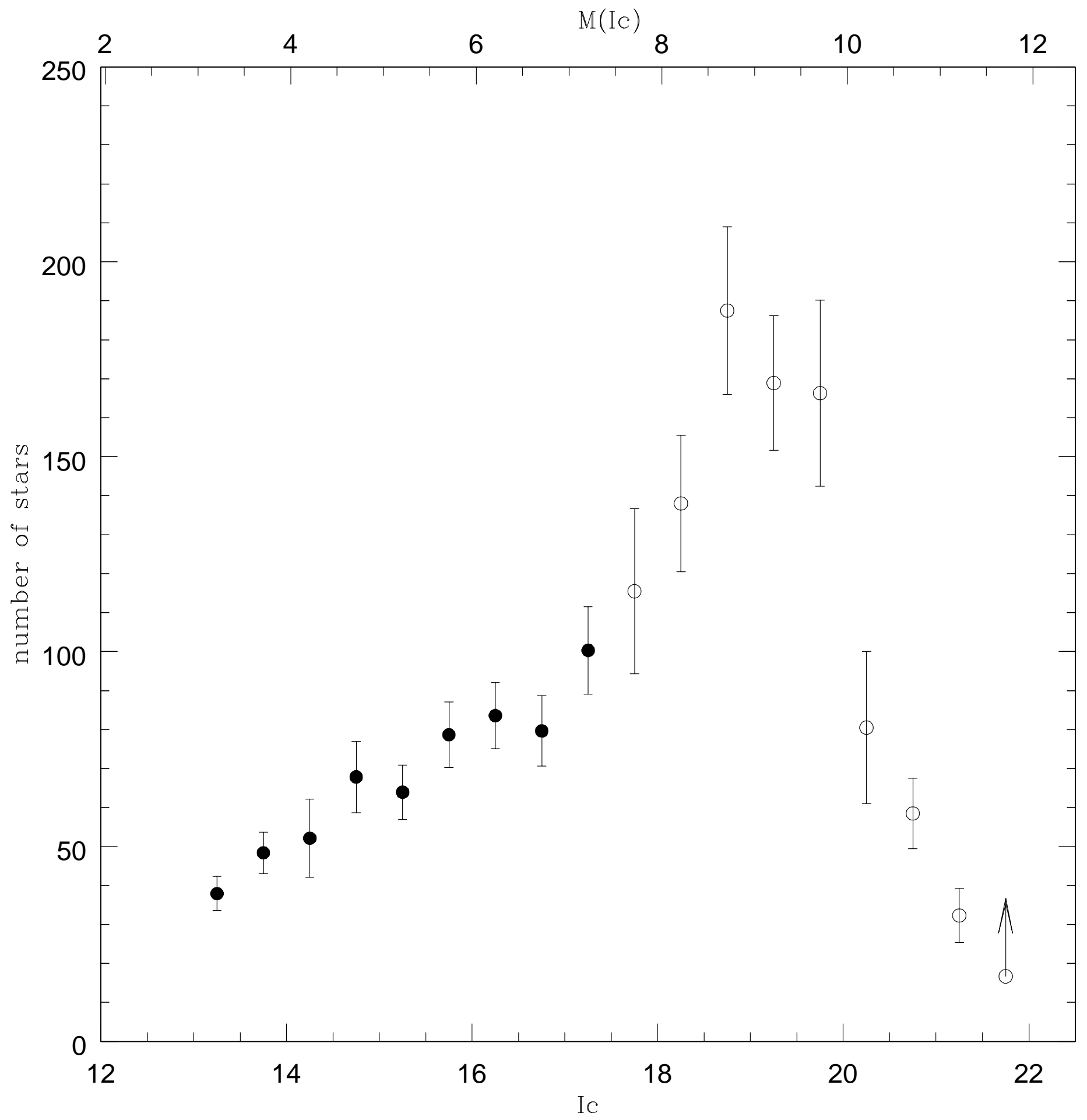




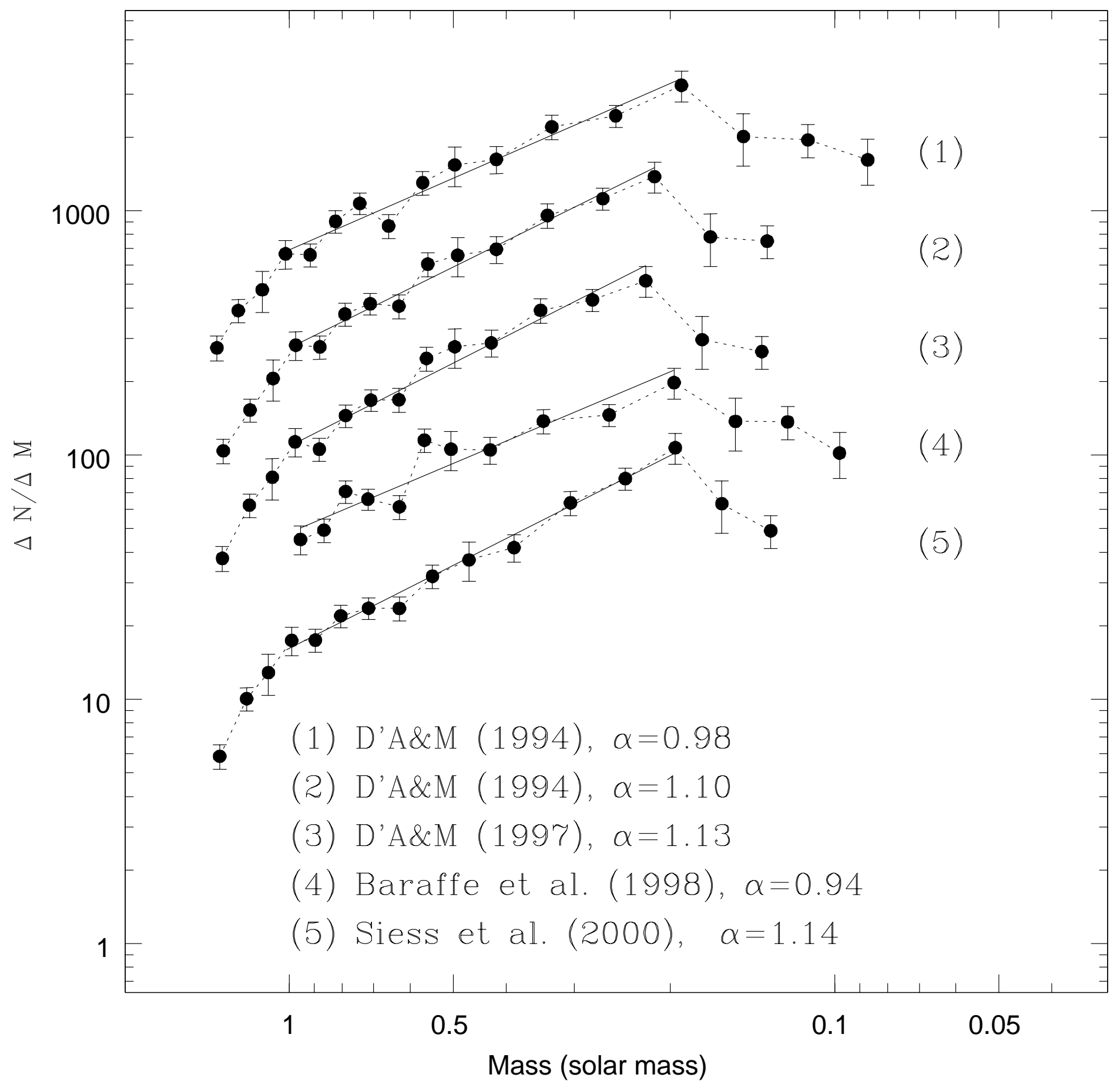




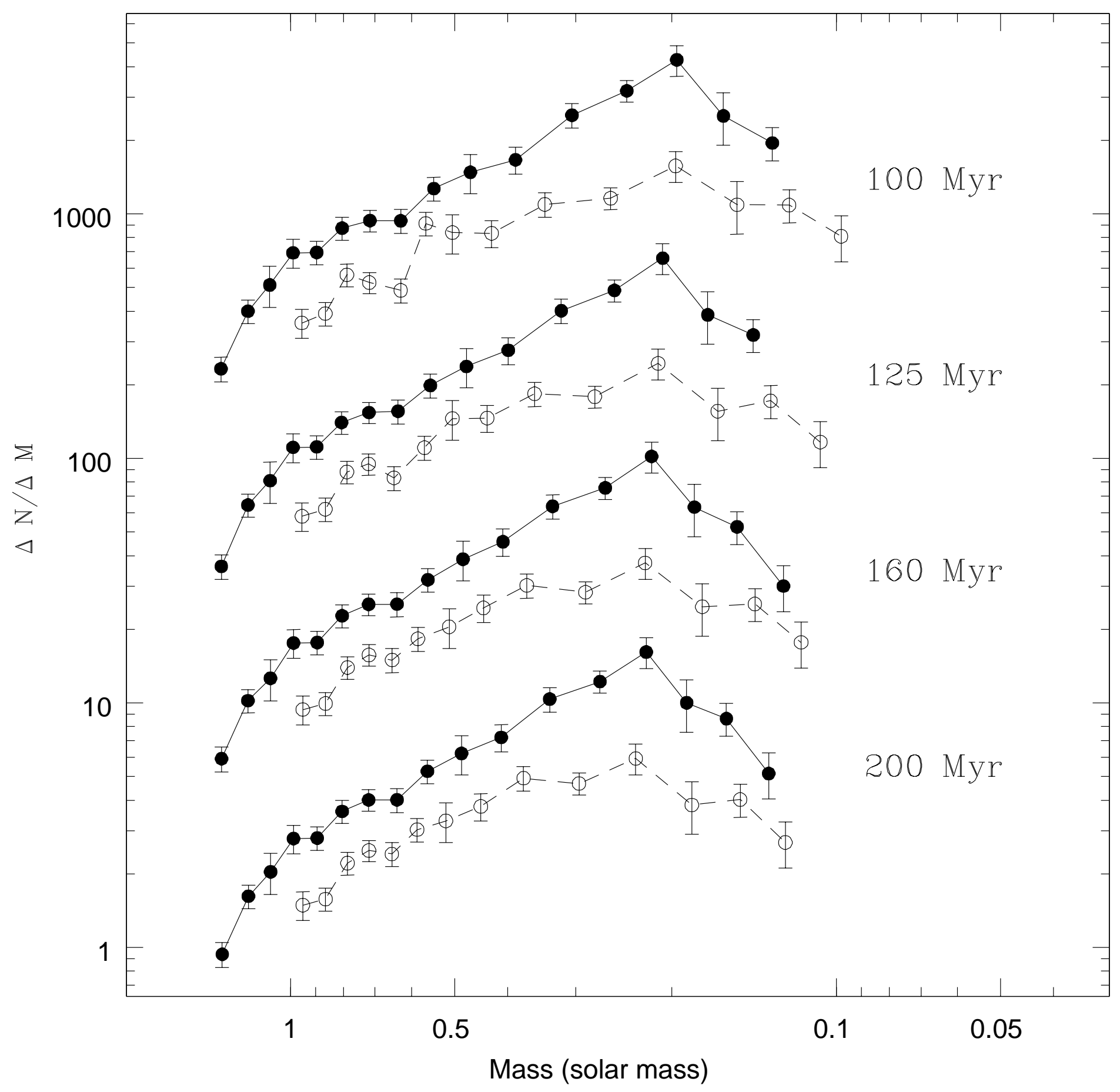




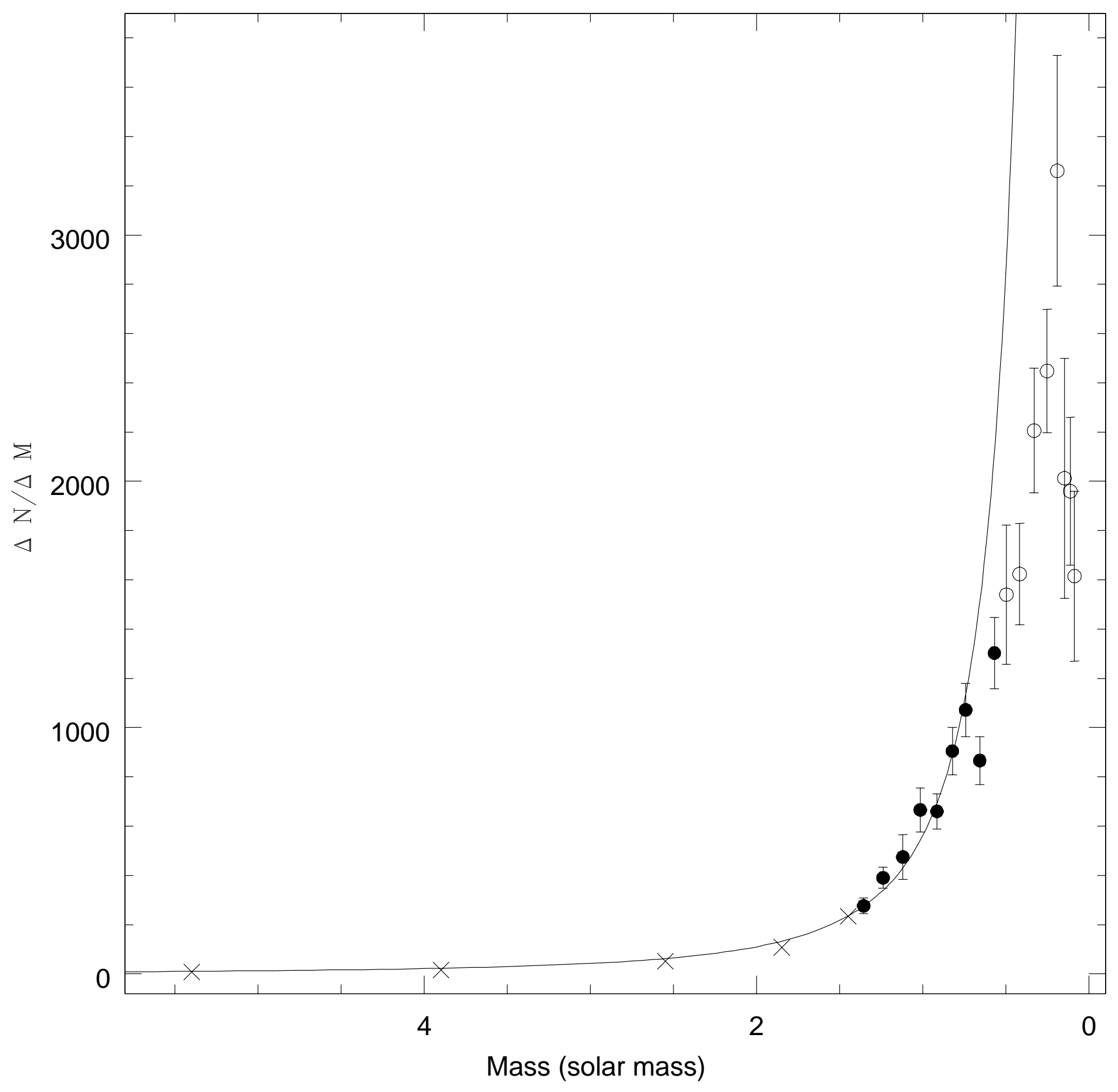




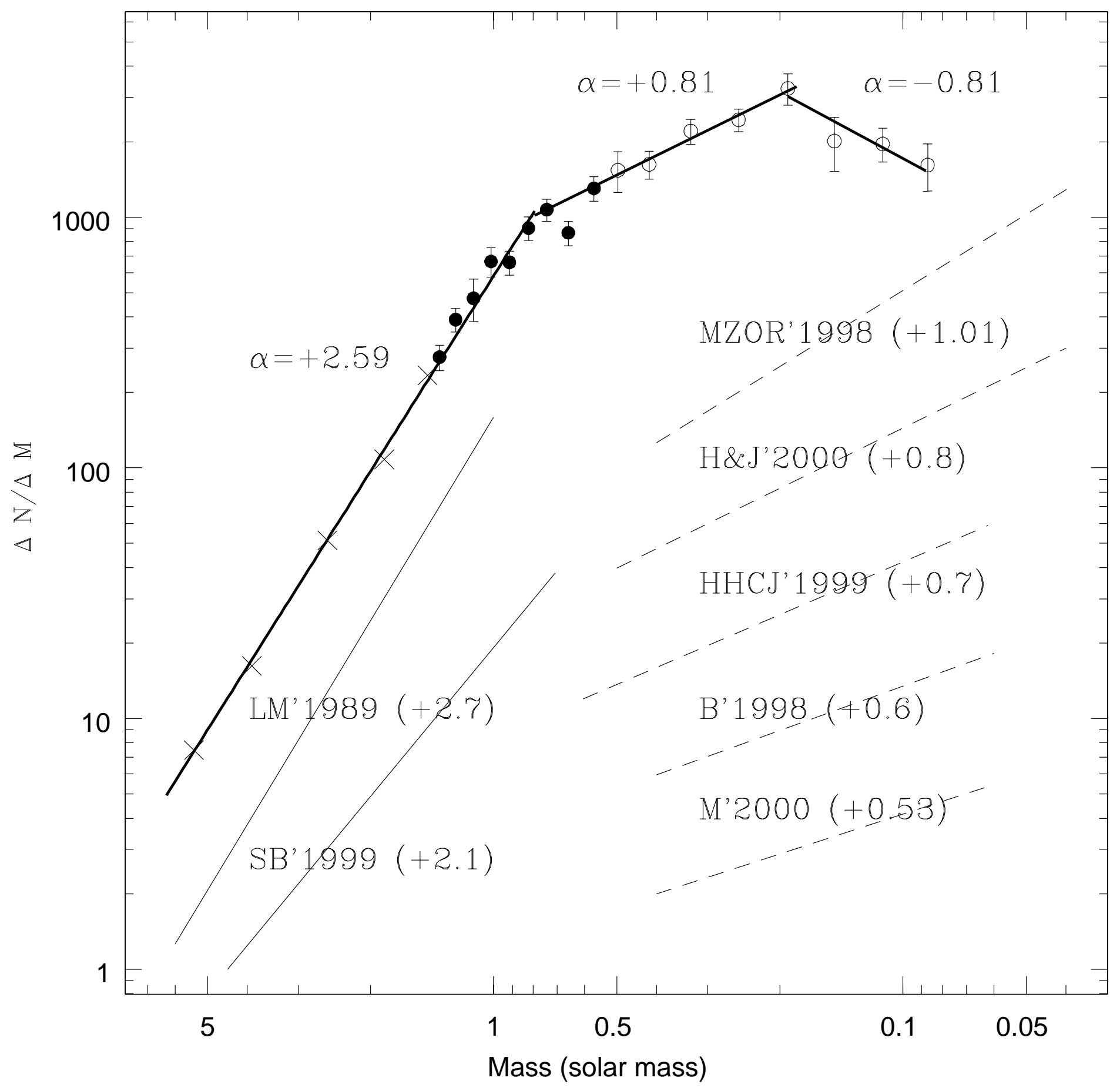




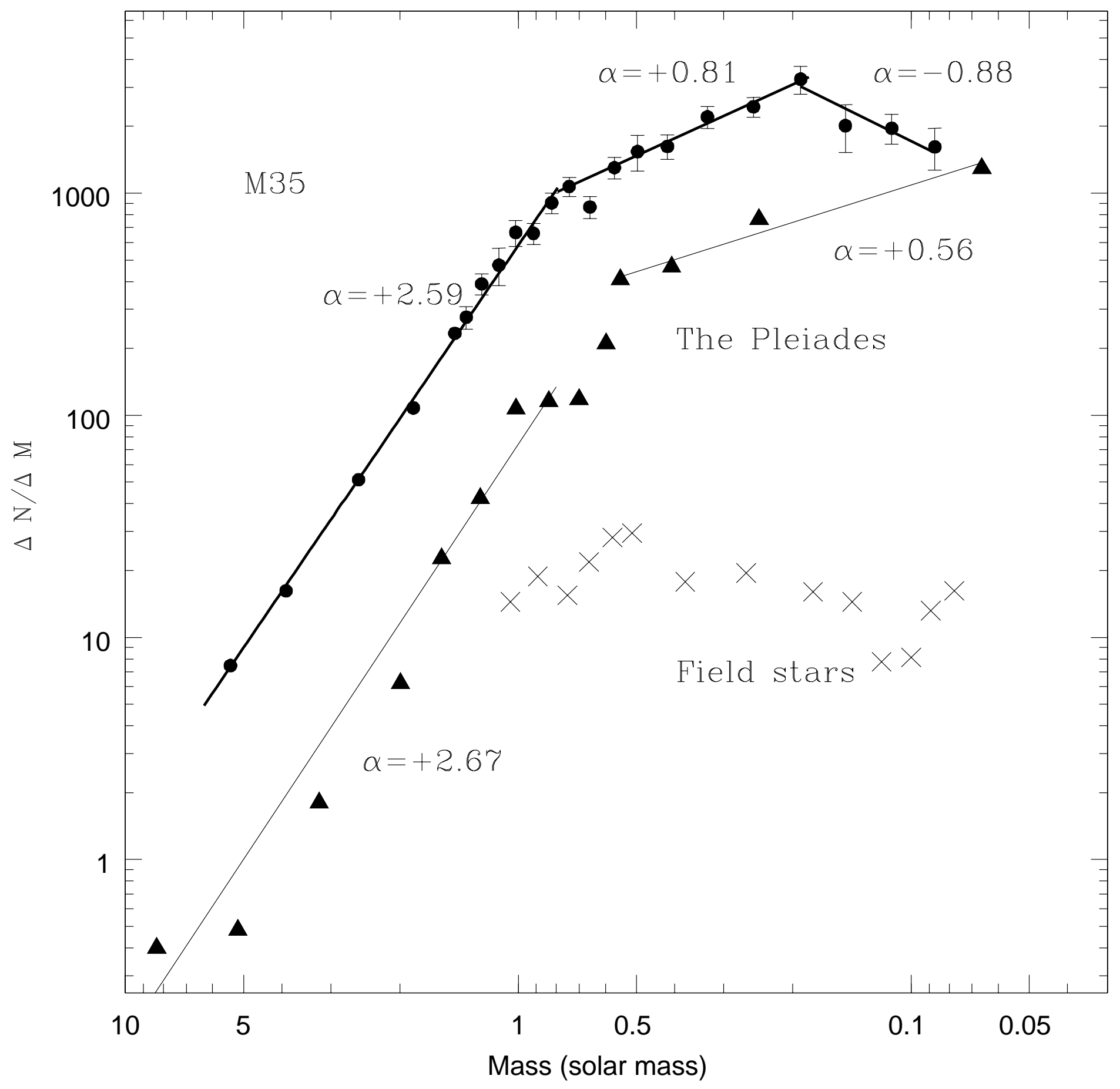




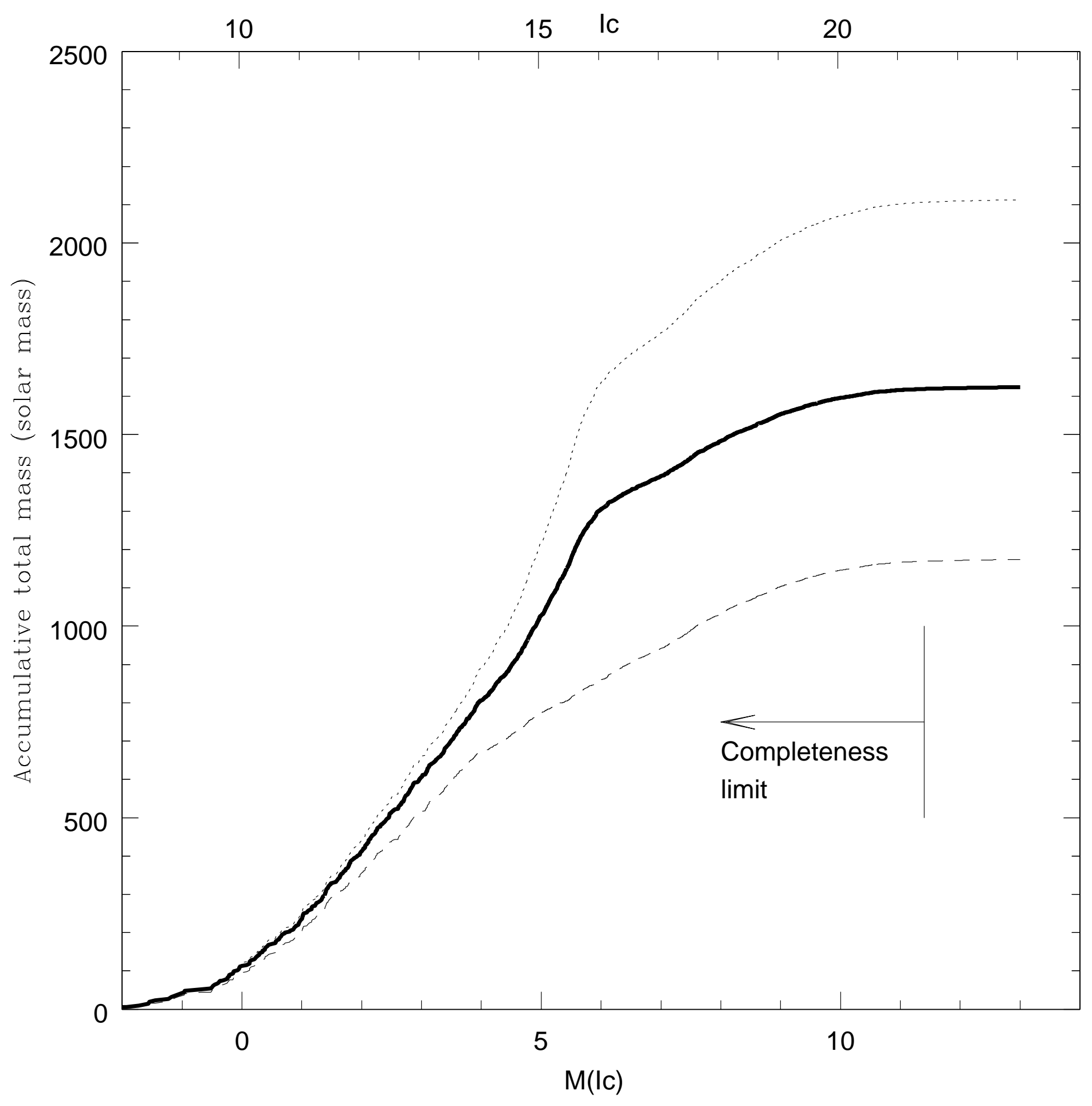




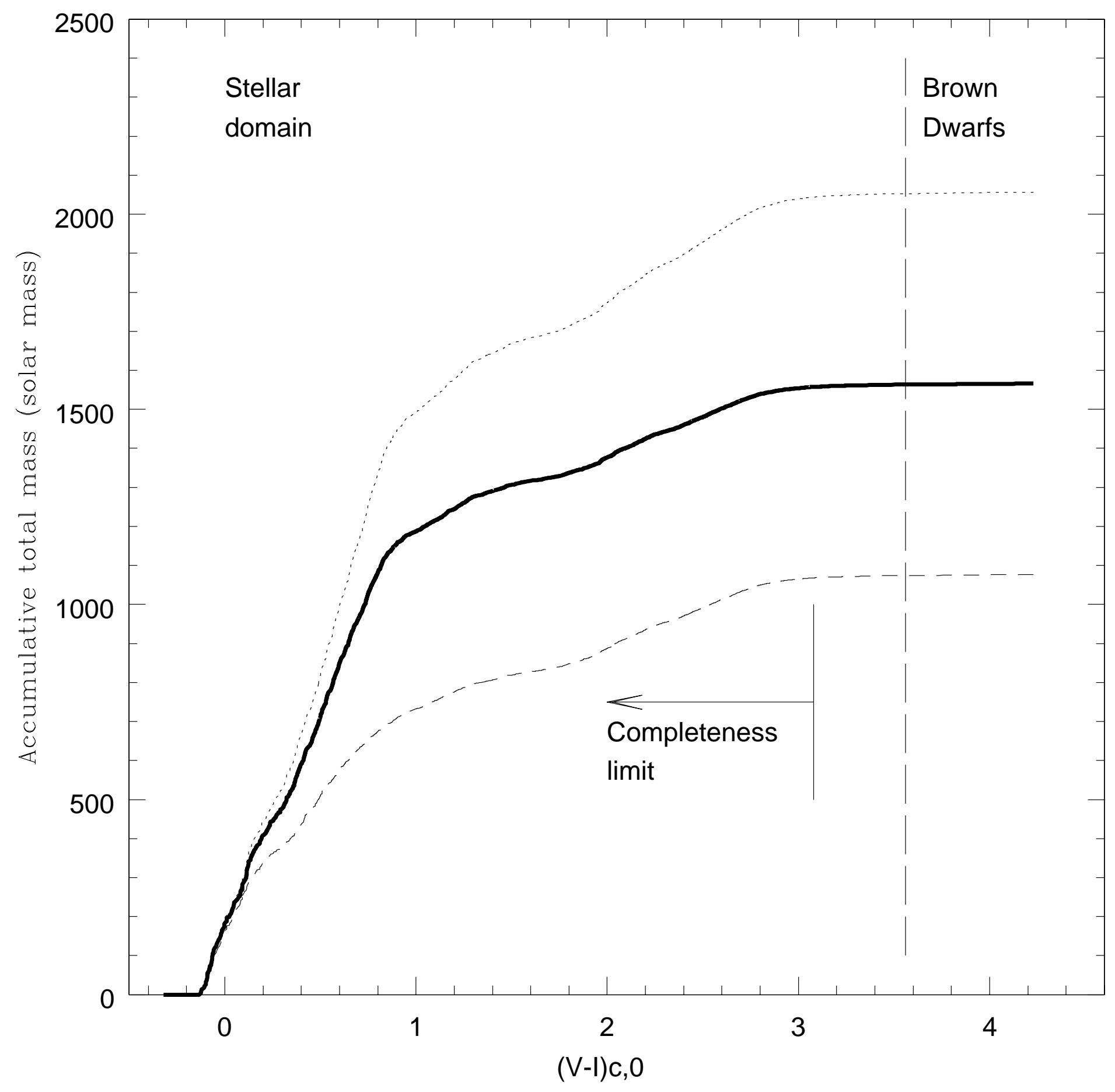




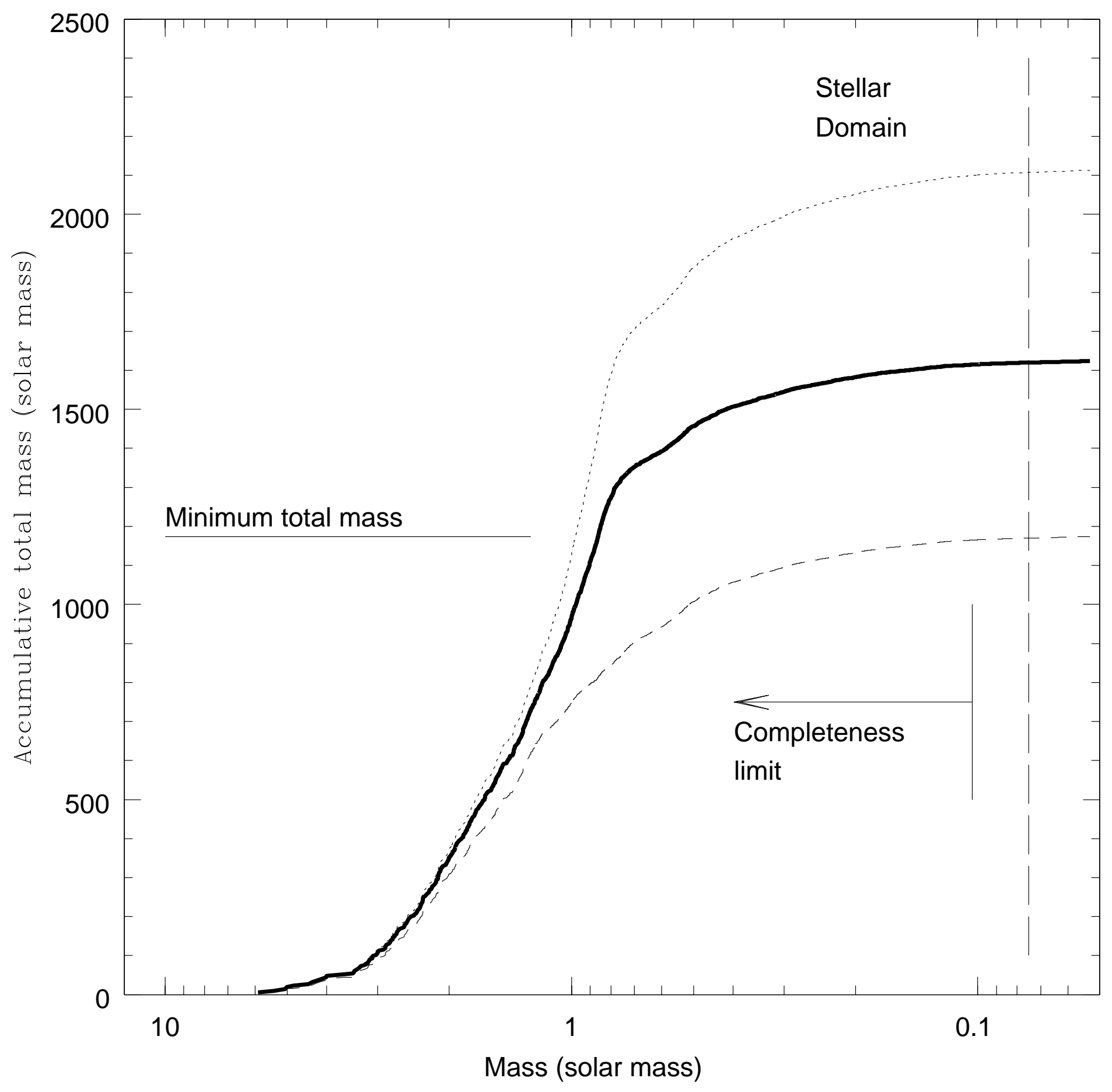


Table 2: M35 candidates (sample). It will be published only in the electronic version. It is available fom the authors upon request.

\begin{tabular}{|c|c|c|c|c|c|c|c|c|c|c|c|c|}
\hline \multirow{2}{*}{ 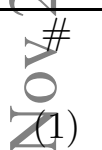 } & $\mathrm{V}$ & $\frac{\mathrm{I}_{c}}{\mathrm{KPNO}}$ & $\left(\mathrm{V}-\mathrm{I}_{c}\right)$ & \multicolumn{2}{|c|}{ CFHT } & \multicolumn{2}{|c|}{$(2000.0)$} & \multirow{2}{*}{$\begin{array}{c}\text { WEBDA } \\
(9)\end{array}$} & \multirow{2}{*}{$\begin{array}{c}\text { MS86 } \\
(10)\end{array}$} & \multirow{2}{*}{$\begin{array}{l}\text { SB99 } \\
(11)\end{array}$} & \multirow{2}{*}{$\begin{array}{l}\text { Prob } \\
(12)\end{array}$} & \multirow{2}{*}{$\begin{array}{c}\text { Member } \\
\text { (13) }\end{array}$} \\
\hline & $(2)$ & $(3)$ & (4) & (5) & $(6)$ & (7) & $(8)$ & & & & & \\
\hline 5001 & 13.561 & 12.737 & 0.824 & - & - & $\begin{array}{lll}69 & 18.49\end{array}$ & 241319.90 & 378 & 349 & 910 & 0.00 & $\mathrm{~N}+$ \\
\hline 5002 & 13.560 & 12.743 & 0.818 & - & - & 6938.20 & 241853.10 & - & - & - & - & Y \\
\hline 5003 & 13.517 & 12.743 & 0.773 & - & - & 6858.51 & 241414.90 & 215 & 223 & 625 & 0.99 & $\mathrm{Y}+$ \\
\hline (.). & …. & $\ldots \ldots$ & $\ldots \ldots$ & ...... & $\ldots \ldots$ & …...... & …........ & $\ldots$ & $\cdots$ & $\cdots$ & $\cdots$ & .. \\
\hline ल..: & $\ldots \ldots$ & ...... & $\cdots \cdots$ & $\cdots \cdots$ & $\cdots \cdots$ & …....... & …........ & $\cdots$ & $\cdots$ & $\cdots$ & $\cdots$ & .. \\
\hline$\ldots$ & $\cdots \cdots$ & $\cdots \cdots$ & $\cdots \cdots$ & $\cdots \cdots$ & 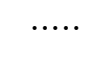 & …....... & ……... & $\cdots$ & $\cdots$ & $\cdots$ & $\cdots$ & .. \\
\hline$\longrightarrow \cdot \cdot$ & $\cdots \cdots$ & $\cdots \cdots$ & $\cdots \cdots$ & $\ldots \ldots$ & $\cdots \cdots$ & …....... & …........ & $\cdots$ & $\cdots$ & $\ldots$ & $\ldots$ &.. \\
\hline Q... & $\cdots \cdots$ & $\cdots \cdots$ & $\cdots \cdots$ & $\cdots \cdots$ & $\cdots \cdots$ & …....... & …........ & $\ldots$ & $\ldots$ & $\ldots$ & $\cdots$ &.. \\
\hline$\ldots$ & $\cdots \cdots$ & $\cdots \cdots$ & $\cdots \cdots$ & $\cdots \cdots$ & $\cdots \cdots$ & ........... & …....... & $\cdots$ & $\cdots$ & $\cdots$ & $\cdots$ & .. \\
\hline 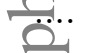 & $\cdots \cdots$ & $\cdots \cdots$ & $\cdots \cdots$ & $\cdots \cdots$ & ..... & .......... & ........... & $\cdots$ & $\cdots$ & $\cdots$ & $\cdots$ & .. \\
\hline$\ldots$ & ...... & ...... & ..... & ...... & ..... & .......... & ............ & $\ldots$ & $\ldots$ & $\cdots$ & .... & .. \\
\hline 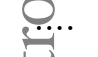 & ...... & $\cdots \cdots$ & $\cdots \cdots$ & $\ldots \ldots$ & $\cdots \cdots$ & …....... & …........ & $\cdots$ & $\cdots$ & $\cdots$ & $\cdots$ &.. \\
\hline 0. & $\cdots \cdots$ & ...... & ..... & ...... & ..... & -......... & …....... & $\cdots$ & $\cdots$ & $\cdots$ & $\cdots$ & .. \\
\hline 6941 & - & - & - & 22.804 & 2.502 & 682.44 & 243157.30 & - & - & - & - & $\mathrm{Y}$ \\
\hline 6942 & - & - & - & 22.826 & 2.315 & 6828.44 & 24339.20 & - & - & - & - & Y \\
\hline ¡6943 & - & - & - & 22.829 & 2.350 & 687.49 & 24832.90 & - & - & - & - & Y \\
\hline 6944 & - & - & - & 22.915 & 2.330 & 6914.50 & 24241.00 & - & - & - & - & $\mathrm{Y}$ \\
\hline-6945 & - & - & - & 23.045 & 2.458 & 6848.99 & 24936.60 & - & - & - & - & Y \\
\hline
\end{tabular}

WEBDA: Open Cluster Database, Mermilliod (1996).

MS86: McNamara \& Sekiguchi (1986).

SB99: Sung \& Bessell (1999). 